\title{
ON THE CONVERGENCE OF THE EXTREMAL EIGENVALUES OF EMPIRICAL COVARIANCE MATRICES WITH DEPENDENCE
}

\author{
DJALIL CHAFAÏ AND KONSTANTIN TIKHOMIROV
}

Dedicated to Alain Pajor \& Nicole Tomczak-Jaegermann

\begin{abstract}
Consider a sample of a centered random vector with unit covariance matrix. We show that under certain regularity assumptions, and up to a natural scaling, the smallest and the largest eigenvalues of the empirical covariance matrix converge, when the dimension and the sample size both tend to infinity, to the left and right edges of the Marchenko-Pastur distribution. The assumptions are related to tails of norms of orthogonal projections. They cover isotropic log-concave random vectors as well as random vectors with i.i.d. coordinates with almost optimal moment conditions. The method is a refinement of the rank one update approach used by Srivastava and Vershynin to produce non-asymptotic quantitative estimates. In other words we provide a new proof of the Bai and Yin theorem using basic tools from probability theory and linear algebra, together with a new extension of this theorem to random matrices with dependent entries.
\end{abstract}

\section{Contents}

1. Introduction

Outline of the argument and novelty

Discussion and extensions

Structure

Notations

2. Proof of Proposition 1.3 and Proposition 1.4

3. Proof of Theorem 1.5

3.1. Feasible lower shift

3.2. Randomization and control of expectations

3.3. Proof of Theorem 1.5, completed

4. Proof of Theorem 1.7

4.1. Feasible upper shift

4.2. Randomization and control of expectations

4.3. Proof of Theorem 1.7, completed

Acknowledgements

References

\section{INTRODUCTION}

Let $\mathbb{N}=\{1,2, \ldots\}$ and let $\left(X_{n}\right)_{n \in \mathbb{N}}$ be a sequence of random vectors where for each $n \in \mathbb{N}$ the random vector $X_{n}$ takes values in $\mathbb{R}^{n}$, is centered, with unit covariance (isotropy):

$$
\mathbb{E}\left(X_{n}\right)=0 \quad \text { and } \quad \mathbb{E}\left(X_{n} \otimes X_{n}\right)=I_{n}
$$

where $I_{n}$ is the $n \times n$ identity matrix. Let $\left(m_{n}\right)_{n \in \mathbb{N}}$ be a sequence in $\mathbb{N}$ such that

$$
0<\underline{\rho}:=\liminf _{n \rightarrow \infty} \frac{n}{m_{n}} \leq \limsup _{n \rightarrow \infty} \frac{n}{m_{n}}=: \bar{\rho}<\infty .
$$

1991 Mathematics Subject Classification. 52A23; 15B52 ; 60B20 ; 62J10.

Key words and phrases. Convex body; Random matrix; covariance matrix; singular value; operator norm; Sherman-Morrison formula; thin-shell inequality; log-concave distribution; dependence. 
For every $n \in \mathbb{N}$, let $X_{n}^{(1)}, \ldots, X_{n}^{\left(m_{n}\right)}$ be i.i.d. copies of $X_{n}$. Their empirical covariance matrix is the $n \times n$ symmetric positive semidefinite random matrix

$$
\widehat{\Sigma}_{n}:=\frac{1}{m_{n}} \sum_{k=1}^{m_{n}} X_{n}^{(k)} \otimes X_{n}^{(k)} .
$$

If $\mathbb{X}_{n}$ denotes the $m_{n} \times n$ rectangular random matrix with i.i.d. rows $X_{n}^{(1)}, \ldots, X_{n}^{\left(m_{n}\right)}$ then

$$
\widehat{\Sigma}_{n}=\frac{1}{m_{n}} \mathbb{X}_{n}^{\top} \mathbb{X}_{n}
$$

Note that $\mathbb{E} \widehat{\Sigma}_{n}=\mathbb{E}\left(X_{n} \otimes X_{n}\right)=I_{n}$. For convenience we define the random matrix

$$
A_{n}:=m_{n} \widehat{\Sigma}_{n}=\mathbb{X}_{n}^{\top} \mathbb{X}_{n}=\sum_{k=1}^{m_{n}} X_{n}^{(k)} \otimes X_{n}^{(k)} .
$$

The eigenvalues of $A_{n}$ are squares of the singular values of $\mathbb{X}_{n}$, and in particular

$$
\lambda_{\max }\left(A_{n}\right)=s_{\max }\left(\mathbb{X}_{n}\right)^{2}=\max _{\|x\|=1}\left\|\mathbb{X}_{n} x\right\|^{2}=\left\|\mathbb{X}_{n}\right\|_{2 \rightarrow 2}^{2} .
$$

When $m_{n} \geq n$ then the smallest eigenvalue of $A_{n}$ satisfies

$$
\lambda_{\min }\left(A_{n}\right)=s_{\min }\left(\mathbb{X}_{n}\right)^{2}=\min _{\|x\|=1}\left\|\mathbb{X}_{n} x\right\|^{2}=\left\|\mathbb{X}_{n}^{-1}\right\|_{2 \rightarrow 2}^{-2}
$$

where the last formula holds only when $\mathbb{X}_{n}$ is invertible (impossible if $m_{n}<n$ ). Above and in the sequel we denote by $\|x\|=\left(x_{1}^{2}+\cdots+x_{n}^{2}\right)^{1 / 2}$ the Euclidean norm of $x \in \mathbb{R}^{n}$.

If $\left(\left(X_{n}\right)_{k}\right)_{1 \leq k \leq n, n \geq 1}$ are i.i.d. standard Gaussians, then the law of the random matrix $\widehat{\Sigma}_{n}$ is known as the real Wishart law, and constitutes a sort of a matrix version of the $\chi^{2}(n)$ law. The law of the eigenvalues of $\widehat{\Sigma}_{n}$ is then called the Laguerre Orthogonal Ensemble, a Boltzmann-Gibbs measure with density on $\left\{\lambda \in[0, \infty)^{m}: \lambda_{1} \geq \cdots \geq \lambda_{n}\right\}$ proportional to

$$
\lambda \mapsto \exp \left(-\frac{m_{n}}{2} \sum_{k=1}^{n} \lambda_{k}+\frac{m_{n}-n+1}{2} \sum_{k=1}^{n} \log \left(\lambda_{n}\right)+\sum_{i<j} \log \left|\lambda_{i}-\lambda_{j}\right|\right),
$$

see [PS11, (7.4.2) p. 192]. This exactly solvable Gaussian model allows to deduce sharp asymptotics for the empirical spectral distribution as well as for the extremal eigenvalues of $\widehat{\Sigma}_{n}$. The famous Marchenko-Pastur theorem [PS11, MP67] states that if

$$
\frac{n}{m_{n}} \underset{n \rightarrow \infty}{\longrightarrow} \rho \text { with } \rho \in(0, \infty)
$$

then almost surely as $n \rightarrow \infty$, the empirical spectral distribution of $\widehat{\Sigma}_{n}$ tends weakly with respect to continuous and bounded test functions to a non-random distribution, namely

$$
\text { a.s. } \frac{1}{m_{n}} \sum_{k=1}^{n} \delta_{\lambda_{k}\left(\widehat{\Sigma}_{n}\right)} \underset{n \rightarrow \infty}{\stackrel{\mathcal{C}_{b}}{\longrightarrow}} \mu_{\rho}
$$

where $\mu_{\rho}$ is the Marchenko-Pastur distibution on $\left[a^{-}, a^{+}\right]$with $a^{ \pm}=(1 \pm \sqrt{\rho})^{2}$ given by

$$
\mu_{\rho}(d x)=\frac{\rho-1}{\rho} \mathbf{1}_{\rho>1} \delta_{0}+\frac{\sqrt{\left(a^{+}-x\right)\left(x-a^{-}\right)}}{\rho 2 \pi x} \mathbf{1}_{\left[a^{-}, a^{+}\right]}(x) d x .
$$

It is a mixture between a Dirac mass at point 0 and an absolutely continuous law. The atom at 0 disappears when $\rho \leq 1$ and is a reminiscence of the rank of $\widehat{\Sigma}_{n}$. The asymptotic phenomenon (1.5) holds beyond the Gaussian case. In particular it was shown by Pajor and Pastur [PP09] that it holds if for every $n \in \mathbb{N}$ the distribution of the isotropic random vector $X_{n}$ is log-concave. Recall that a probability measure $\mu$ on $\mathbb{R}^{n}$ with density $\varphi$ is log-concave when $\varphi=e^{-V}$ with $V$ convex, see [Bor74, BGVV14. Log-concavity allows some kind of geometric dependence but imposes sub-exponential tails. The asympotitic phenomenon (1.5) also holds if $\left(\left(X_{n}\right)_{k}\right)_{1 \leq k \leq n, n \geq 1}$ are i.i.d. with finite second moment 
[BS10, PS11]. An extension to various other models can be found in Bai and Zhou [BZ08], Pastur and Shcherbina [PS11], Adamczak [Ada11], and Adamczak and Chafaï [AC15].

The weak convergence (1.5) does not provide much information at the edge on the behavior of the extremal atoms, and what one can actually extract from (1.5) is that

$$
\text { a.s. } \quad \limsup _{n \rightarrow \infty} \lambda_{\min }\left(\widehat{\Sigma}_{n}\right) \leq(1-\sqrt{\rho})^{2} \leq(1+\sqrt{\rho})^{2} \leq \liminf _{n \rightarrow \infty} \lambda_{\max }\left(\widehat{\Sigma}_{n}\right)
$$

where the first inequality is considered only in the case where $m_{n} \geq n$. If $\left(\left(X_{n}\right)_{k}\right)_{1 \leq k \leq n, n \geq 1}$ are i.i.d. with finite fourth moment then it was shown by Bai and Yin BY88, $\overline{\text { YBK}}$ 88, BY93 using combinatorics that the convergence in (1.6) holds:

$$
\text { a.s. } \quad(1-\sqrt{\rho})^{2}=\lim _{n \rightarrow \infty} \lambda_{\min }\left(\widehat{\Sigma}_{n}\right) \leq \lim _{n \rightarrow \infty} \lambda_{\max }\left(\widehat{\Sigma}_{n}\right)=(1+\sqrt{\rho})^{2},
$$

where the first equality is considered only in the case where $m_{n} \geq n$. The convergence of the largest eigenvalue in the right hand side of (1.7) does not take place if $\left(\left(X_{n}\right)_{k}\right)_{1 \leq k \leq n, n \geq 1}$ are i.i.d. with infinite fourth moment, see [BS10]. It was understood recently that the convergence of the smallest eigenvalue in the left hand side of (1.7) holds actually as soon as $\left(\left(X_{n}\right)_{k}\right)_{1 \leq k \leq n, n \geq 1}$ are i.i.d. with finite second moment, see Tikhomirov [Tik15].

An analytic proof of (1.7) based on the resolvent is also available, and we refer to Bordenave [Bor14 for the i.i.d. case, and to Bai and Silverstein [BS98], Pillai and Yin [PY14, and Richard and Guionnet [RG14] for more sophisticated models still not including the case in which the law of $X_{n}$ is $\log$-concave for every $n \in \mathbb{N}$. Note that the analytic approach was also used for various models of random matrices by Haagerup and Thorbjørnsen [HT05, Schultz [Sch05, and by Capitaine and Donati-Martin CDM07.

The study of quantitative high dimensional non-asymptotic properties of the smallest and of the largest eigenvalues of empirical covariance matrices was the subject of an intense line of research in the recent years; in connection with log-concavity, see for instance Adamczak, Litvak, Pajor, and Tomczak-Jaegermann [ALPTJ10, ALPTJ11, Rudelson and Vershynin [RV10, Koltchinskii and Mendelson [KM15, and references therein.

Non-asymptotic estimates for (1.7) were obtained by Srivastava and Vershynin SV13] using a rank one update strategy which takes advantage of the decomposition (1.4). This approach is an elementary interplay between probability and linear algebra, which is remarkably neither analytic nor combinatorial. The outcome is that with high probability

$$
\left(1-c^{-} \sqrt{\frac{n}{m_{n}}}\right)^{2} \leq \lambda_{\min }\left(\widehat{\Sigma}_{n}\right) \leq \lambda_{\max }\left(\widehat{\Sigma}_{n}\right) \leq\left(1+c^{+} \sqrt{\frac{n}{m_{n}}}\right)^{2},
$$

where the first inequality is considered only in the case where $m_{n} \geq n$. Here $c^{ \pm}>0$ are constants, and thus one cannot deduce (1.7) from (1.8). The approach of Srivastava and Vershynin is a randomization of the spectral sparsification method developed by Batson, Spielman, and Srivastava BSS09, BSS12]; the idea of using rank one updates can also be found in the works of Benaych-Georges and Nadakuditi [BGN12]. This approach requires the control of tails of norms of projections of $X_{n}$, a condition which is satisfied by logconcave distributions thanks to the thin-shell phenomenon. This condition is also satisfied if $\left(\left(X_{n}\right)_{k}\right)_{1 \leq k \leq n, n \geq 1}$ are i.i.d. with a finite moment of order $2+\varepsilon$ for the lower bound on the smallest eigenvalue in (1.7) and i.i.d. with a finite moment of order $4+\varepsilon$ for the upper bound on the largest eigenvalue in (1.7). This method was also used recently by Yaskov Yas14, Yas15.

Our main results below lie between the original Bai-Yin theorem and the more recent work of Srivastava and Vershynin. Our contribution is to show that the non-asymptotic approach of Srivastava and Vershynin is indeed suitable to prove and extend the sharp Bai-Yin theorem, which is an asymptotic result, under fairly general asumptions on tails of norms of projections of $X_{n}$, which allow heavy tailed i.i.d. as well as log-concavity! When the coordinates of $X_{n}$ are i.i.d. our approach reaches the (almost) optimal moment 
condition: finite second moment for the smallest eigenvalue and finite fourth moment for the largest.

Our results are based on the following tail conditions on the norm of projections.

Definition 1.1 (Weak Tail Projection property (WTP)). Let $X_{n}, n \in \mathbb{N}$, be as in (1.1). We say that the Weak Tail Projection (WTP) property holds when the following is true:

(a) The family $\left(\left\langle X_{n}, y\right\rangle^{2}\right)_{n \in \mathbb{N}, y \in S^{n-1}}$ is uniformly integrable, in other words

$$
\lim _{M \rightarrow \infty} \sup _{\substack{n \in \mathbb{N} \\ y \in S^{n-1}}} \mathbb{E}\left(\left\langle X_{n}, y\right\rangle^{2} \mathbf{1}_{\left\{\left\langle X_{n}, y\right\rangle^{2} \geq M\right\}}\right)=0,
$$

where $S^{n-1}:=\left\{y \in \mathbb{R}^{n}:\|y\|=1\right\}$ denotes the unit sphere of $\mathbb{R}^{n}$;

(b) There exist two functions $f: \mathbb{N} \rightarrow[0,1]$ and $g: \mathbb{N} \rightarrow \mathbb{R}_{+}$such that $f(r) \rightarrow 0$ and $g(r) \rightarrow 0$ as $r \rightarrow \infty$ and for every $n \in \mathbb{N}$ and any orthogonal projection $\mathrm{P}: \mathbb{R}^{n} \rightarrow \mathbb{R}^{n}$ with $\mathrm{P} \neq 0$

$$
\mathbb{P}\left(\left\|\mathrm{P} X_{n}\right\|^{2}-\operatorname{rankP} \geq f(\operatorname{rankP}) \operatorname{rankP}\right) \leq g(\operatorname{rankP}) .
$$

This can be less formally written as $\mathbb{P}\left(\left\|\mathrm{P} X_{n}\right\|^{2}-r \geq o(r)\right) \leq o(1)$ where $r:=\operatorname{rankP}$ and where the "o" are with respect to $r$ and are uniform in $n$.

Definition 1.2 (Strong Tail Projection property (STP)). Let $X_{n}, n \in \mathbb{N}$, be as in (1.1). We say the Strong Tail Projection (STP) property holds when there exist $f: \mathbb{N} \rightarrow[0,1]$ and $g: \mathbb{N} \rightarrow \mathbb{R}_{+}$such that $f(r) \rightarrow 0$ and $g(r) \rightarrow 0$ as $r \rightarrow \infty$, and for every $n \in \mathbb{N}$, for any orthogonal projection $\mathrm{P}: \mathbb{R}^{n} \rightarrow \mathbb{R}^{n}$ with $\mathrm{P} \neq 0$, for any real $t \geq f(\operatorname{rankP}) \operatorname{rankP}$ we have

$$
\mathbb{P}\left(\left\|\mathrm{P} X_{n}\right\|^{2}-\operatorname{rankP} \geq t\right) \leq \frac{g(\operatorname{rankP}) \operatorname{rankP}}{t^{2}} .
$$

This can be less formally written as $\mathbb{P}\left(\left\|\mathrm{P} X_{n}\right\|^{2}-r \geq t\right) \leq o(r) t^{-2}$ where $t \geq o(r)$ and $r:=\operatorname{rankP}$ and where the "o" are with respect to $r$ and are uniform in $n$.

Note that $\mathbb{E}\left(\left\|\mathrm{P} X_{n}\right\|^{2}\right)=\operatorname{rankP}$ since $X_{n}$ is isotropic. The properties (WTP) and (STP) were inspired by the "strong regularity assumption" used by Srivastava and Vershynin SV13. They are specially designed to obtain a sharp Bai-Yin type asymptotic result in the i.i.d. case with (almost) optimal moment assumptions, as well as in the log-concave case.

It is easy to see that (STP) implies that for any $\varepsilon>0$, the $4-\varepsilon$ moments of 1-dimensional marginals of $X_{n}$ 's are uniformly bounded; in particular, in this case the vectors $\left(X_{n}\right)_{n \in \mathbb{N}}$ satisfy condition (WTP-a $)$. Next, condition (WTP-b) is clearly weaker than (STP $)$; thus, (STP) implies (WTP), hence the qualifiers "Strong" and "Weak" for these properties.

Proposition 1.3 below, proved in Section 2, implies that if $\left(X_{n}\right)_{n \in \mathbb{N}}$ is as in (1.1) and if $X_{n}$ is $\log$-concave for every $n \in \mathbb{N}$ then properties (WTP) and (STP) are satisfied. It is a consequence of the thin-shell and sub-exponential tails phenomena for these distributions.

Proposition 1.3 (Log-concave random vectors). Let $X_{n}, n \in \mathbb{N}$, be as in (1.1), and suppose that the centered isotropic random vector $X_{n}$ is log-concave for any $n \in \mathbb{N}$. Then a stronger form of (STP) holds with

$$
\left\{\left\|\mathrm{P} X_{n}\right\|^{2}-\operatorname{rankP} \geq t\right\} \quad \text { replaced by } \quad\left\{\left|\left\|\mathrm{P} X_{n}\right\|^{2}-\operatorname{rankP}\right| \geq t\right\} .
$$

The next proposition implies that if $\left(\left(X_{n}\right)_{k}\right)_{1<k \leq n, n \in \mathbb{N}}$ are i.i.d. then property (WTP) holds, and if moreover these i.i.d. random variables have finite fourth moment then property (STP) holds.

Proposition 1.4 (Random vectors with i.i.d. coordinates). Let $X_{n}, n \in \mathbb{N}$, be as in (1.1), and suppose that the coordinates $\left(\left(X_{n}\right)_{k}\right)_{1 \leq k \leq n, n \in \mathbb{N}}$ are i.i.d. distributed as a real random variable $\xi$ with zero mean and unit variance. Then, denoting $r:=\operatorname{rankP}$, 
- a stronger version of (WTP) holds, with, in (WTP-b),

$$
\left\{\left\|\mathrm{P} X_{n}\right\|^{2}-r \geq f(r) r\right\} \quad \text { replaced by } \quad\left\{\left|\left\|\mathrm{P} X_{n}\right\|^{2}-r\right| \geq f(r) r\right\} ;
$$

- if moreover $\mathbb{E}\left(\xi^{4}\right)<\infty$ then a stronger version of (STP) holds, with

$$
\left\{\left\|\mathrm{P} X_{n}\right\|^{2}-r \geq t\right\} \quad \text { replaced by } \quad\left\{\left|\left\|\mathrm{P} X_{n}\right\|^{2}-r\right| \geq t\right\} .
$$

Our main results are stated in the following couple of theorems and corollaries.

Theorem 1.5 (Smallest eigenvalue). Let $X_{n}, m_{n}$, and $A_{n}, n \in \mathbb{N}$, be as in (1.1), (1.2), and (1.4) respectively. If $\bar{\rho}<1$ (in particular $m_{n}>n$ for $n \gg 1$ ), and if (WTP-a) and (WTP-b) are satisfied then

$$
\liminf _{n \rightarrow \infty} \frac{\mathbb{E}\left(\lambda_{\min }\left(A_{n}\right)\right)}{\left(\sqrt{m_{n}}-\sqrt{n}\right)^{2}} \geq 1
$$

Theorem [1.5] is proved in Section 3 .

Combining Theorem 1.5 with Proposition 1.3 and Proposition 1.4, we obtain the following corollary. The second part, which is the Bai-Yin edge convergence (1.7) of the smallest eigenvalue in probability, is obtained by combining the first part of the corollary with the Marchenko-Pastur bound (1.6) for the smallest eigenvalue.

Corollary 1.6 (Smallest eigenvalue convergence). Let $X_{n}, m_{n}, \widehat{\Sigma}_{n}$, and $A_{n}, n \in \mathbb{N}$, be as in (1.1), (1.2), (1.3), and (1.4) respectively. If $\bar{\rho}<1$ (in particular $m_{n}>n$ for $n \gg 1)$ and if the centered isotropic random vector $X_{n}$ is log-concave for every $n \in \mathbb{N}$ or if $\left(\left(X_{n}\right)_{k}\right)_{1 \leq k \leq n, n \in \mathbb{N}}$ are i.i.d. then

$$
\liminf _{n \rightarrow \infty} \frac{\mathbb{E}\left(\lambda_{\min }\left(A_{n}\right)\right)}{\left(\sqrt{m_{n}}-\sqrt{n}\right)^{2}} \geq 1
$$

If additionally $\lim _{n \rightarrow \infty} \frac{n}{m_{n}}=\rho$ with $\rho \in(0,1)$, in other words $\underline{\rho}=\bar{\rho} \in(0,1)$, then

$$
\lambda_{\min }\left(\widehat{\Sigma}_{n}\right) \underset{n \rightarrow \infty}{\stackrel{\mathbb{P}}{\longrightarrow}}(1-\sqrt{\rho})^{2} .
$$

Theorem 1.7 (Largest eigenvalue). Let $X_{n}, m_{n}$, and $A_{n}, n \in \mathbb{N}$, be as in (1.1), (1.2), and (1.4) respectively. If (STP) holds then

$$
\limsup _{n \rightarrow \infty} \frac{\mathbb{E}\left(\lambda_{\max }(A)\right)}{\left(\sqrt{m_{n}}+\sqrt{n}\right)^{2}} \leq 1 .
$$

Theorem 1.7 is proved in Section 4 .

Combining Theorem [1.7 with Proposition 1.3 and Proposition 1.4, we obtain the following statement (again, for the second part we use the Marchenko-Pastur law):

Corollary 1.8 (Largest eigenvalue convergence). Let $X_{n}, m_{n}, \widehat{\Sigma}_{n}$ and $A_{n},, n \in \mathbb{N}$, be as in (1.1), (1.2), (1.3), and (1.4) respectively. If the centered isotropic random vector $X_{n}$ is log-concave for every $n \in \mathbb{N}$ or if $\left(\left(X_{n}\right)_{k}\right)_{1 \leq k \leq n, n \in \mathbb{N}}$ are i.i.d. with finite 4 th moment then

$$
\limsup _{n \rightarrow \infty} \frac{\mathbb{E}\left(\lambda_{\max }\left(A_{n}\right)\right)}{\left(\sqrt{m_{n}}+\sqrt{n}\right)^{2}} \leq 1 \text {. }
$$

If additionally $\lim _{n \rightarrow \infty} \frac{n}{m_{n}}=\rho$ with $\rho \in(0, \infty)$ in other words $\underline{\rho}=\bar{\rho} \in(0, \infty)$, then

$$
\lambda_{\max }\left(\widehat{\Sigma}_{n}\right) \underset{n \rightarrow \infty}{\stackrel{\mathbb{P}}{\rightarrow}}(1+\sqrt{\rho})^{2} .
$$


Outline of the argument and novelty. Assume we are given a random matrix

$$
A=\sum_{k=1}^{m} X^{(k)} \otimes X^{(k)}
$$

where $X^{(k)}$ are i.i.d. isotropic random vectors. As we already mentioned above, the key ingredient in estimating the extremal eigenvalues of $A$ is the following rank one update formula known as the Sherman-Morrison formula:

$$
\left(M+x x^{\top}\right)^{-1}=M^{-1}-\frac{M^{-1} x x^{\top} M^{-1}}{1+x^{\top} M^{-1} x},
$$

which is valid for any non-singular $n \times n$ matrix $M$ and a vector $x$ with $1+x^{\top} M^{-1} x \neq 0$. Using the above identity and assuming that $M$ is symmetric, the restriction on $\mathbb{R}$ of the Cauchy-Stieltjes transform of the spectral distribution of $M+x x^{T}$, which is defined as an appropriately scaled trace of $\left(u-M-x x^{\top}\right)^{-1}, u \in \mathbb{R}$, can be in a simple way expressed in terms of the Cauchy-Stieltjes transform of $M$. To be more precise, setting

$$
A^{(0)}:=0 \quad \text { and } \quad A^{(k)}:=A^{(k-1)}+X^{(k)} \otimes X^{(k)}, \quad k=1, \ldots, m,
$$

we get, for any $k=1, \ldots, m$ and any $u \in \mathbb{R}$,

$$
\operatorname{tr}\left(u-A^{(k)}\right)^{-1}=\operatorname{tr}\left(u-A^{(k-1)}\right)^{-1}+\frac{X^{(k)^{\top}}\left(u-A^{(k-1)}\right)^{-2} X^{(k)}}{1-X^{(k)^{\top}}\left(u-A^{(k-1)}\right)^{-1} X^{(k)}} .
$$

A crucial observation, made already in [BSS09] and further developed in [SV13] is that the Cauchy-Stieltjes transform on the real line can be efficiently used to control the extreme eigenvalues of the matrix. The basic idea is, starting from some fixed $u_{0} \neq 0$, to define inductively a sequence of random numbers $u_{k}(k \leq m)$ in such a way that all $u_{k}$ 's stay on the same side of the spectra of $A^{(k)}$ 's, at the same time not departing too far from the spectrum. Then the expectation of the corresponding extreme eigenvalue of $A=A^{(m)}$ can be estimated by $\mathbb{E} u_{m}$. The increments $u_{k}-u_{k-1}$ are defined with help of the last formula as certain functions of $A^{(k-1)}, u_{k-1}$ and $X^{(k)}$, and their expectations are controlled using the information on the distribution of $X^{(k)}$ as well as certain induction hypothesis. At this level, the approach used in the present paper is similar to SV13.

On the other hand, as our result is asymptotically sharp and covers the i.i.d. case with almost optimal moment conditions, the technical part of our argument differs significantly from [SV13]. In particular, we introduce the "regularity shifts", which are designed in such a way that $u_{k}$ 's stay "sufficiently far" from the spectrum of $A^{(k)}$ 's, which guarantees validity of certain concentration inequalities, whereas at the same time not departing "too far" so that one still gets a satisfactory estimate of the expectation of the spectral edges. The shifts (which we denote by $\delta_{R}^{k}$ and $\Delta_{R}^{k}$ ) are defined in Sections 3.3 and 4.3 (see, in particular, lemmas 3.5 and 4.7).

Let us emphasize once more that the proofs we obtain are much simpler and shorter than the original combinatorial approach of Bai-Yin and the analytic approach based on the resolvent (more precisely the Cauchy-Stieltjes transform on the complex plane outside the real axis), while the class of distributions covered in our paper is much larger. In particular, Theorem 1.5] of the present paper essentially recovers a recent result [Tik15]. In must be noted, however, that in our paper we replace convergence almost surely with the weaker convergence in probability.

Discussion and extensions. In this note we restrict our analysis to random vectors with real coordinates because we think that this is simply more adapted to geometric dependence such as log-concavity. It is likely that the method remains valid for random vectors with complex entries. The Bai-Yin theorem is also available for random symmetric matrices (which are the sum of rank two updates which are no longer positive semidefinite) 
but it is unclear to us if one can adapt the method to this situation. One can ask in another direction if the method remains usable for non-white population covariance matrices, and for the so-called information plus noise covariance matrices, two models studied at the edge by Bai and Silverstein BS98, BS12 among others. One can ask finally if the method allows to extract at least the scaling of the Tracy-Widom fluctuation at the edge. For the Tracy-Widom fluctuation at the edge of empirical covariance matrices we refer to Johansson [Joh00], Johnstone [Joh01, Borodin and Forrester [BF03, Soshnikov [Sos02], Péché [Péc09], Feldheim and Sodin [FS10, Pillai and Yin [PY14], and references therein. It was shown by Lee and Yin LY14 that for centered Wigner matrices the finiteness of the fourth moment is more than enough for the Tracy-Widom fluctuation of the largest eigenvalue. One can ask the same for the largest eigenvalue of the empirical covariance matrix of random vectors with i.i.d. entries, and one can additionally ask if a finite second moment is enough for the Tracy-Widom fluctuation of the smallest eigenvalue.

Structure. The rest of the article is structured as follows. Section 2 provides the proof of Proposition 1.3 and Proposition [1.4. In Sections 3 and 4 we prove Theorem 1.5$]$ and Theorem [1.7, respectively.

Notations. We set $\|v\|:=\sqrt{v_{1}+\cdots+v_{n}^{2}}$ and $\|v\|_{\infty}:=\max \left(\left|v_{1}\right|, \ldots,\left|v_{n}\right|\right)$ for any vector $v \in \mathbb{R}^{n}$. We denote $\|f\|_{\infty}:=\sup _{x \in S}|f(x)|$ for any function $f: S \rightarrow \mathbb{R}$. We often use the notation $|S|:=\operatorname{card}(S)$ for a set $S$. Further, we denote by

$$
\lambda_{\max }(M):=\lambda_{1}(M) \geq \cdots \geq \lambda_{n}(M)=: \lambda_{\min }(M)
$$

the eigenvalues of a symmetric $n \times n$ matrix $M \in \mathcal{M}_{n}(\mathbb{R})$. We denote by $I_{n}$ the $n \times$ $n$ identity matrix. For any real number $u$ we sometimes abridge $u I_{n}$ into $u$ in matrix expressions such as in $M-u I_{n}=M-u$.

\section{Proof of Proposition 1.3 and Proposition 1.4}

Proof of Proposition 1.3. Assume $X$ is a centered isotropic log-concave vector in $\mathbb{R}^{n}$ and $\mathrm{P}: \mathbb{R}^{n} \rightarrow \mathbb{R}^{n}$ is a non-zero orthogonal projection. The random vector $\mathrm{P} X$ is log-concave with mean zero and covariance matrix $\mathrm{PP}^{\top}=\mathrm{P}^{2}=\mathrm{P}$. Restricted to the image of $\mathrm{P}$, the vector $Y=\mathrm{P} X$ is log-concave with covariance matrix $I_{r}$ where $r=\operatorname{rankP}$. The so-called thin-shell phenomenon ABP03 states that "most" of the distribution of $Y$ is supported in a thin-shell around the Euclidean sphere of radius $\sqrt{r}$. Quantitative estimates have been obtained notably by Klartag [Kla07, Fleury [Fle10, Guédon and Milman [GM11, see also Guédon Gué14. On the other hand, it is also known that the tail of the of norm of $Y$ is sub-exponenial, see Paouris [Pao06], and also Admaczak et al [ALL ${ }^{+}$]. The following inequality, taken from GM11, Theorem 1.1], captures both phenomena: there exist absolute constants $c, C \in(0, \infty)$ such that for any real $u \geq 0$,

$$
\mathbb{P}(|||Y||-\sqrt{r}| \geq u \sqrt{r}) \leq C \exp \left(-c \sqrt{r} \min \left(u, u^{3}\right)\right) .
$$

This is more than enough for our needs. Namely, let $\beta \in(0,1 / 20)$, and let $u=u(r) \in$ $(0, \infty)$ and $\alpha=\alpha(r) \in(0,1)$ be such that $\alpha \geq(1+r)^{-\beta}$ and $u \geq \max \left((1+r)^{-\beta}, 2 \alpha /(1-\right.$ $\left.\left.\alpha^{2}\right)\right)$. Note that $2 \alpha /\left(1-\alpha^{2}\right) \in(0,1)$ when $\alpha \in(0, \sqrt{2}-1)$, and that $2 \alpha /\left(1-\alpha^{2}\right) \rightarrow 0$ as $\alpha \rightarrow 0$. Now, using the inequality $\exp (-2 t) \leq t^{-4}$ for $t>0$, we get, if $\alpha u \in(0,1]$,

$$
\begin{aligned}
\mathbb{P}\left(\|Y\|^{2}-r \geq u^{2} r\right) & =\mathbb{P}\left(\|Y\| \geq \sqrt{r+u^{2} r}\right) \\
& \leq \mathbb{P}(\|Y\| \geq \sqrt{r}+\alpha u \sqrt{r}) \\
& \leq C \exp \left(-c \sqrt{r}(\alpha u)^{3}\right) \\
& \leq \frac{2^{4} C}{c^{4}\left(u^{2} r\right)^{2} \alpha^{12} u^{8}}=\frac{o(r)}{\left(u^{2} r\right)^{2}},
\end{aligned}
$$


while if $\alpha u \in[1, \infty)$ we get

$$
\mathbb{P}\left(\|Y\|^{2}-r \geq u^{2} r\right) \leq \cdots \leq C \exp (-c \sqrt{r} \alpha u) \leq \frac{2^{4} C}{c^{4}\left(u^{2} r\right)^{2} \alpha^{4}}=\frac{o(r)}{\left(u^{2} r\right)^{2}} .
$$

Similarly, for an arbitrary $\beta \in(0,1 / 20)$, let $u=u(r) \in(0, \infty)$ and $\alpha=\alpha(r) \in(0,1)$ be such that $\alpha \geq(1+r)^{-\beta}$ and $u \geq \max \left((1+r)^{-\beta}, 2 \alpha /\left(1+\alpha^{2}\right)\right)$. If $u>1$ then necessarily

$$
\mathbb{P}\left(\|Y\|^{2}-r \leq-u^{2} r\right)=0 .
$$

Otherwise, $\alpha u \in(0,1]$ and using again the inequality $\exp (-2 t) \leq t^{-4}$ for $t>0$, we get

$$
\begin{aligned}
\mathbb{P}\left(\|Y\|^{2}-r \leq-u^{2} r\right) & \leq \mathbb{P}\left(\|Y\| \leq \sqrt{r-u^{2} r}\right) \\
& \leq \mathbb{P}(\|Y\| \leq \sqrt{r}-\alpha u \sqrt{r}) \\
& \leq C \exp \left(-c \sqrt{r}(\alpha u)^{3}\right) \\
& \leq \frac{2^{4} C}{c^{4}\left(u^{2} r\right)^{2} \alpha^{12} u^{8}}=\frac{o(r)}{\left(u^{2} r\right)^{2}} .
\end{aligned}
$$

Thus, we obtain $\mathbb{P}\left(\left|\|Y\|^{2}-r\right| \geq t\right) \leq o(r) t^{-2}$ for $t \geq o(r)$ as expected.

Proof of Proposition 1.4.

- Proof of the first part (uniform integrability (WTP-a)). Recall that we are given a random variable $\xi$ with zero mean and unit variance and that for every $n \in \mathbb{N}$ the coordinates of $X_{n}$ are independent copies of $\xi$. We want to show that

$$
\lim _{M \rightarrow \infty} \sup _{\substack{n \in \mathbb{N} \\ y \in S^{n-1}}} \mathbb{E}\left(\left\langle X_{n}, y\right\rangle^{2} \mathbf{1}_{\left\{\left\langle X_{n}, y\right\rangle^{2} \geq M\right\}}\right)=0 .
$$

For every $x \in \mathbb{R}^{n}$, we define $f_{n}(x):=\left\langle X_{n}, x\right\rangle$. Clearly, $\mathbb{E}\left(f_{n}^{2}(x)\right)=\|x\|^{2}$ since $X_{n}$ is isotropic. Let us start with some comments to understand the problem. The random variables $\left(f_{n}^{2}(y)\right)_{n \in \mathbb{N}, y \in S^{n-1}}$ belong to the unit sphere of $L^{1}$. If $\xi$ has finite fourth moment $B:=\mathbb{E}\left(\xi^{4}\right)<\infty$ then by expanding and using isotropy we get $\mathbb{E}\left(f_{n}^{4}(y)\right) \leq \max (B, 3)$ which shows that the family $\left(f_{n}^{2}(y)\right)_{n \in \mathbb{N}, y \in S^{n-1}}$ is bounded in $L^{2}$ and thus uniformly integrable. How to proceed when $\xi$ does not have a finite fourth moment? If $y$ belongs to the canonical basis of $\mathbb{R}^{n}$ then $f_{n}(y)$ is distributed as $\xi$ and has thus the same integrability. On the other hand, if $y$ is far from being sparse, say $y=\left(n^{-1 / 2}, \ldots, n^{-1 / 2}\right)$, then $f_{n}(y)$ is distributed as $n^{-1 / 2}\left(\xi_{1}+\cdots+\xi_{n}\right)$ where the $\xi_{i}$ 's are independent copies of $\xi$, which is close in distribution to the standard Gaussian law $\mathcal{N}(0,1)$ by the Central Limit Theorem (CLT).

We will use the following uniform quantitative CLT. Even though it probably exists somewhere in the literature, we provide a short proof for convenience. It can probably also be proved using the classical Fourier analytic smoothing inequality [Fel71, equation (3.13) XVI.3 p. 538] which is the basis of many uniform CLT estimates.

Lemma 2.1 (Uniform quantitative CLT). Let $\xi$ be a random variable with zero mean and unit variance and let $\Phi$ be the cumulative distribution of the standard real Gaussian law $\mathcal{N}(0,1)$ of zero mean and unit variance. For any $\varepsilon>0$ there exists $\delta>0$ depending only on $\varepsilon$ and the law of $\xi$ with the following property: if $n \in \mathbb{N}$ and $y \in S^{n-1}$ is such that $\|y\|_{\infty} \leq \delta$, then the cumulative distribution function $F_{n}$ of $\sum_{i=1}^{n} y_{i} \xi_{i}$ satisfies

$$
\left\|F_{n}-\Phi\right\|_{\infty} \leq \varepsilon
$$


Proof of Lemma 2.1. To prove the lemma, let us assume the contrary. Then there exists $\varepsilon>0$ and a sequence $\left(y_{m}\right)_{m \geq 1}$ in $\ell^{2}(\mathbb{N})$ such that $\left\|y_{m}\right\|=1$ and $\left\|y_{m}\right\|_{\infty} \leq$ $1 / m$ for every $m \in \mathbb{N}$, and such that $\left\|F_{m}-\Phi\right\|_{\infty}>\varepsilon$ where $F_{m}$ is the cumulative distribution function of $S_{m}:=\sum_{i=1}^{\infty} y_{m, i} \xi_{i}$. Let $\varphi_{m}$ be the characteristic function of $S_{m}$. We have $\varphi_{m}(t)=\prod_{i=1}^{\infty} \varphi\left(y_{m, i} t\right)$ where $\varphi$ is the characteristic function of $\xi$. Fix any $t \in \mathbb{R}$. By assumption on $\xi$, we get $\varphi(t)=1-\frac{t^{2}}{2}+o\left(t^{2}\right)$. Hence, using the identities $\left\|y_{m}\right\|^{2}:=\sum_{i=1}^{\infty} y_{m, i}^{2}=1$ and $\left\|y_{m}\right\|_{\infty}:=\max _{i \in \mathbb{N}}\left|y_{m, i}\right| \leq 1 / m$ together with the formula (valid for $m \rightarrow \infty$ ):

$\varphi_{m}(t)=\prod_{i=1}^{\infty}\left(1-\frac{y_{m, i}^{2} t^{2}}{2}+o\left(\left(y_{m, i}^{2} t^{2}\right)\right)\right)=\exp \left(-\frac{t^{2}}{2} \sum_{i=1}^{\infty} y_{m, i}^{2}+\sum_{i=1}^{\infty} o\left(y_{m, i}^{2} t^{2}\right)\right)$,

we get $\lim _{m \rightarrow \infty} \varphi_{m}(t)=e^{-t^{2} / 2}$. By the Lévy theorem for characteristic functions, it follows that $F_{m} \rightarrow \Phi$ pointwise as $m \rightarrow \infty$, which yields $S_{m} \rightarrow \mathcal{N}(0,1)$ weakly as $m \rightarrow \infty$, contradicting to our initial assumption.

Let us continue with the proof of the uniform integrability. Since $\xi^{2} \in L^{1}$ we get, by dominated convergence,

$$
h(M):=\mathbb{E}\left(\xi^{2} \mathbf{1}_{\left\{\xi^{2} \geq M\right\}}\right) \underset{M \rightarrow \infty}{\longrightarrow} 0 .
$$

Let $\varepsilon>0$, and let $\delta>0$ be defined from $\varepsilon$ and the law of $\xi$ as in the above lemma (we can, of course, assume that $\delta \rightarrow 0$ with $\varepsilon \rightarrow 0$ ). Let $M>0$ and $n \in \mathbb{N}$ and $y \in S^{n-1}$. Let us write $y=w+z$ where $w_{i}:=y_{i} \mathbf{1}_{\left|y_{i}\right| \leq \delta^{2}}$ and $z_{i}:=y_{i} \mathbf{1}_{\left|y_{i}\right|>\delta^{2}}$ for any $i=1, \ldots, n$. Then it is easily seen that

- $\operatorname{supp}(z) \cap \operatorname{supp}(w)=\varnothing$;

- $\|w\| \leq 1$ and $\|w\|_{\infty} \leq \delta^{2}$;

- $\|z\| \leq 1$ and $|\operatorname{supp}(z)| \leq 1 / \delta^{4}$,

where $\operatorname{supp}(x):=\left\{i: x_{i} \neq 0\right\}$. Now we have $f_{n}^{2}(y) \leq 2\left(f_{n}^{2}(w)+f_{n}^{2}(z)\right)$ and

$$
\begin{aligned}
\mathbb{E}\left(f_{n}^{2}(y) \mathbf{1}_{\left\{f_{n}^{2}(y) \geq M\right\}}\right) \leq & 2 \mathbb{E}\left(\left(f_{n}^{2}(w)+f_{n}^{2}(z)\right)\left(\mathbf{1}_{\left\{f_{n}^{2}(w) \geq \frac{M}{4}\right\}}+\mathbf{1}_{\left\{f_{n}^{2}(z) \geq \frac{M}{4}\right\}}\right)\right) \\
\leq & 2\|z\|^{2} \mathbb{P}\left(f_{n}^{2}(w) \geq \frac{1}{4} M\right)+2\|w\|^{2} \mathbb{P}\left(f_{n}^{2}(z) \geq \frac{1}{4} M\right) \\
& +2 \mathbb{E}\left(f_{n}^{2}(z) \mathbf{1}_{\left\{f_{n}^{2}(z) \geq \frac{M}{4}\right\}}\right)+2 \mathbb{E}\left(f_{n}^{2}(w) \mathbf{1}_{\left\{f_{n}^{2}(w) \geq \frac{M}{4}\right\}}\right) \\
= & (*)+(* *) \\
& +(* * *)+(* * * *) .
\end{aligned}
$$

Now, by Markov's inequality

$$
(*)+(* *) \leq \frac{16}{M} .
$$

Second, using that $f_{n}^{2}(x) \leq\left\|\mathrm{P}_{x} X_{n}\right\|^{2}$, valid for any $x \in \mathbb{R}^{n}$ with $\|x\| \leq 1$ and orthogonal projection $\mathrm{P}_{x}$ onto its support, we obtain

$$
\begin{aligned}
(* * *) & \leq 2 \mathbb{E}\left(\left(\sum_{i: z_{i} \neq 0} \xi_{i}^{2}\right) \sum_{i: z_{i} \neq 0} \mathbf{1}_{\left\{\xi_{i}^{2} \geq \frac{\delta^{8} M}{4}\right\}}\right) \\
& =2 \mathbb{E}\left(\sum_{i, j \in \operatorname{supp}(z)} \xi_{i}^{2} \mathbf{1}_{\left\{\xi_{j}^{2} \geq \frac{\delta^{8} M}{4}\right\}}\right) \\
& \leq 2\left(\frac{1}{\delta^{8}} \frac{4}{\delta^{8} M}+\frac{h\left(\frac{\delta^{8} M}{4}\right)}{\delta^{4}}\right)=\frac{8}{\delta^{16} M}+\frac{2 h\left(\frac{\delta^{8} M}{4}\right)}{\delta^{4}},
\end{aligned}
$$

where in the third line we used Markov's inequality. Third, we write

$$
(* * * *)=2 \mathbb{E}\left(f_{n}^{2}(w)\right)-2 \mathbb{E}\left(f_{n}^{2}(w) \mathbf{1}_{\left\{f_{n}^{2}(w)<\frac{M}{4}\right\}}\right) .
$$


Now if $\|w\| \leq \delta$ then $(* * * *) \leq 2 \delta^{2}$. Suppose in contrast that $\|w\|>\delta$, and denote $w_{*}:=w /\|w\|$. Then $\left\|w_{*}\right\|_{\infty} \leq \delta$, and therefore, by the CLT of Lemma 2.1, the distribution of $f_{n}\left(w_{*}\right)$ is $\varepsilon$-close to $\mathcal{N}(0,1)$, and in particular, there exist $M_{*}(\varepsilon)>0$ and $\rho(\varepsilon)>0$ depending only on $\varepsilon$ such that $\rho(\varepsilon) \rightarrow 0$ as $\varepsilon \rightarrow 0$ and

$$
\mathbb{E}\left(f_{n}^{2}\left(w_{*}\right) \mathbf{1}_{\left\{f_{n}^{2}\left(w_{*}\right)<M_{*}(\varepsilon)\right\}}\right) \geq 1-\rho(\varepsilon) .
$$

Thus, having in mind that $f_{n}^{2}\left(w_{*}\right)=f_{n}^{2}(w) /\|w\|^{2}$, we get

$$
(* * * *) \leq 2\|w\|^{2} \rho(\varepsilon) \leq 2 \rho(\varepsilon)
$$

provided that $M \geq 4 M_{*}(\varepsilon)$. So, in all cases, as long as $M$ is sufficiently large,

$$
(* * * *) \leq 2 \delta^{2}+2 \rho(\varepsilon) .
$$

Finally, take any $M>0$ such that $M \geq \max \left(16 / \varepsilon, 8 /\left(\delta^{16} \varepsilon\right), 4 M_{*}(\varepsilon)\right)$ and such that $2 h\left(\delta^{8} M / 4\right) / \delta^{14} \leq \varepsilon$; then the desired result follows from

$$
(*)+(* *)+(* * *)+(* * * *) \leq 4 \varepsilon+2 \delta^{2}+2 \rho(\varepsilon) .
$$

- Proof of the first part (improved ( $(\overline{\mathrm{WTP}-\mathrm{b}})$ ). As before, we assume that $\xi$ is a random variable with zero mean and unit variance, and denote by $\left(\xi_{i}\right)$ a sequence of i.i.d. copies of $\xi$. Let us recall a kind of the weak law of large numbers for weighted sums, taken from [Tik15, Lemma 5], which can be seen as a consequence of Lévy's continuity theorem for characteristic functions: if $\left(\eta_{i}\right)_{i \in I}$ is a sequence (finite or infinite) of i.i.d. real random variables with zero mean then for every $\varepsilon>0$ there exists $\delta>0$ which may depend on the law of the random variables with the following property: for every deterministic sequence $\left(t_{i}\right)_{i \in I}$ in $[0, \infty)$ such that $\|t\|_{1}:=\sum_{i \in I} t_{i}=1$ and $\|t\|_{\infty}:=\max _{i \in I} t_{i} \leq \delta$, we have

$$
\mathbb{P}\left(\left|\sum_{i \in I} t_{i} \eta_{i}\right|>\varepsilon\right)<\varepsilon
$$

Setting $\eta_{i}:=\xi_{i}^{2}-1$, it follows that there exists $h:(0,1] \rightarrow(0,1]$ such that, given any $\varepsilon>0$ and a sequence $\left(t_{i}\right)_{i \in I}$ in $[0, \infty)$ with $\|t\|_{1}=1$ and $\|t\|_{\infty} \leq h(\varepsilon)$, we have

$$
\mathbb{P}\left(\left|\sum_{i \in I} t_{i} \xi_{i}^{2}-1\right|>\varepsilon\right) \leq \varepsilon
$$

Without loss of generality, one can take $h$ strictly monotone (i.e. invertible).

We now proceed similarly to [SV13, Proposition 1.3]. Fix $n \in \mathbb{N}$ and let P be a non-zero orthogonal projection of $\mathbb{R}^{n}$ of rank $r$. Let $X=\left(\xi_{k}\right)_{1 \leq k \leq n}$ be a random vector of $\mathbb{R}^{n}$ with $\xi_{1}, \ldots, \xi_{n}$ i.i.d. copies of $\xi$. We have

$$
\|\mathrm{P} X\|^{2}=\langle X, \mathrm{P} X\rangle \text {. }
$$

Let us also denote the matrix of $\mathrm{P}$ in the canonical basis as $\mathrm{P}$. We have $P^{2}=P=$ $P^{\top}$ and $\operatorname{tr}(\mathrm{P})=\operatorname{rank}(\mathrm{P})=r$. Let $\mathrm{P}_{0}$ be the matrix obtained from $\mathrm{P}$ by zeroing the diagonal. We have $\mathrm{P}-\mathrm{P}_{0}=\operatorname{diag}(\mathrm{P})$. A standard decoupling inequality proved in [Ver11] (see also the book [dlPG99]) states that for any convex function $F$,

$$
\mathbb{E}\left(F\left(\left\langle X, \mathrm{P}_{0} X\right\rangle\right)\right) \leq \mathbb{E}\left(F\left(4\left\langle X, \mathrm{P}_{0} X^{\prime}\right\rangle\right)\right),
$$

where $X^{\prime}$ is an independent copy of $X$. In particular the choice $F(u)=u^{2}$ gives

$$
\mathbb{E}\left(\left\langle X, \mathrm{P}_{0} X\right\rangle^{2}\right) \leq 16 \mathbb{E}\left(\left\langle X, \mathrm{P}_{0} X^{\prime}\right\rangle^{2}\right)
$$


Now recall that if $Z$ is a random vector of $\mathbb{R}^{n}$ with covariance matrix $\Gamma$ and if $B$ is a $n \times n$ matrix then $\mathbb{E}(\langle Z, B Z\rangle)=\operatorname{tr}\left(\Gamma B^{\top}\right)$. Seeing $X$ and $X^{\prime}$ as column vectors,

$$
\begin{aligned}
\mathbb{E}\left(\left\langle X, \mathrm{P}_{0} X^{\prime}\right\rangle^{2}\right) & =\mathbb{E}\left(X^{\prime \top} \mathrm{P}_{0} X X^{\top} \mathrm{P}_{0} X^{\prime}\right) \\
& =\mathbb{E}\left(X^{\prime \top} \mathrm{P}_{0} \mathbb{E}\left(X X^{\top}\right) \mathrm{P}_{0} X^{\prime}\right) \\
& =\mathbb{E}\left(X^{\prime \top} \mathrm{P}_{0}^{2} X^{\prime}\right) \\
& =\operatorname{tr}\left(\mathrm{P}_{0}^{2}\right) \\
& \leq 2 \operatorname{tr}\left(\left(\mathrm{P}-\mathrm{P}_{0}\right)^{2}\right)+2 \operatorname{tr}\left(\mathrm{P}^{2}\right) \\
& \leq 2 \operatorname{tr}\left(\mathrm{P}^{2}\right)+2 \operatorname{tr}\left(\mathrm{P}^{2}\right) \\
& =4 r .
\end{aligned}
$$

Therefore

$$
\mathbb{E}\left(\left\langle X, \mathrm{P}_{0} X\right\rangle^{2}\right) \leq 64 r
$$

and by Markov's inequality we get

$$
\mathbb{P}\left(\left|\left\langle X, \mathrm{P}_{0} X\right\rangle\right|>r^{3 / 4}\right) \leq \frac{64}{\sqrt{r}}
$$

Next note that

$$
\left\langle X,\left(\mathrm{P}-\mathrm{P}_{0}\right) X\right\rangle=\sum_{i=1}^{n} \mathrm{P}_{i, i} \xi_{i}^{2}
$$

with $0 \leq P_{i, i} \leq 1$ and $\sum_{i=1}^{n} P_{i, i}=r$. Hence taking $t_{i}=P_{i, i} / r$ and $\varepsilon:=h^{-1}(1 / r)$ with $\varepsilon:=1$ if $1 / r$ is outside of the range of $h$, we get, using (2.1),

$$
\mathbb{P}\left(\left|\left\langle X,\left(\mathrm{P}-\mathrm{P}_{0}\right) X\right\rangle-r\right|>\varepsilon r\right) \leq \varepsilon .
$$

Finally, by combining with (2.3) we obtain

$$
\mathbb{P}\left(|\langle X, \mathrm{P} X\rangle-r|>\left(\varepsilon+r^{-1 / 4}\right) r\right) \leq \varepsilon+\frac{64}{\sqrt{r}}
$$

and this implies the desired result, namely

$$
\mathbb{P}(|\langle X, \mathrm{P} X\rangle-r|>o(r))=o(1) .
$$

- Proof of the second part (improved $(\overline{\mathrm{STP}})$ ). Let $\xi$ be a random variable with zero mean, unit variance and a finite fourth moment. Further, let $\mathrm{P}=\left(\mathrm{P}_{i j}\right), \mathrm{P}_{0}$ and $r$ have the same meaning as before, and $X=\left(\xi_{k}\right)_{1 \leq k \leq n}$, where $\xi_{k}$ 's are i.i.d. copies of $\xi$. For any $u>0$ we have

$$
\begin{aligned}
& \mathbb{P}\left(\left|\|\mathrm{P} X\|^{2}-r\right|>u\right) \\
& \quad \leq \mathbb{P}\left(\left|\left\langle X, \mathrm{P}_{0} X\right\rangle\right|>u / 2\right)+\mathbb{P}\left(\left|\left\langle X,\left(\mathrm{P}-\mathrm{P}_{0}\right) X\right\rangle-r\right|>u / 2\right) \\
& \quad=:(*)+(* *) .
\end{aligned}
$$

Let us estimate the last two quantities $(*)$ and $(* *)$ separately. In both cases we will compute selected moments and use Markov's inequality. Set $B:=\mathbb{E}\left(\xi_{k}^{4}\right)$. Note that $B \geq 1$ since $\mathbb{E} \xi^{2}=1$. Since $\xi_{k}$ 's are independent, we get, for any unit vector $y=\left(y_{k}\right)_{1 \leq k \leq n}$,

$$
\mathbb{E}\left(\langle X, y\rangle^{4}\right)=\sum_{i} \mathbb{E}\left(\xi_{i}^{4}\right) y_{i}^{2} y_{i}^{2}+3 \sum_{i \neq j} \mathbb{E}\left(\xi_{i}^{2}\right) \mathbb{E}\left(\xi_{j}^{2}\right) y_{i}^{2} y_{j}^{2} \leq \max (B, 3) .
$$

The decoupling inequality (2.2) with $F(u)=u^{4}$ gives

$$
\mathbb{E}\left(\left\langle X, \mathrm{P}_{0} X\right\rangle^{4}\right) \leq 256 \mathbb{E}\left(\left\langle X, \mathrm{P}_{0} X^{\prime}\right\rangle^{4}\right),
$$


where $X^{\prime}$ is an independent copy of $X$. Next, in view of (2.4), we get

$$
\mathbb{E}\left(\left\langle X, \mathrm{P}_{0} X^{\prime}\right\rangle^{4}\right) \leq \mathbb{E}\left(\left\|\mathrm{P}_{0} X^{\prime}\right\|^{4}\right) \max _{\|y\|=1} \mathbb{E}\left(\langle X, y\rangle^{4}\right) \leq \max (B, 3) \mathbb{E}\left(\left\|\mathrm{P}_{0} X^{\prime}\right\|^{4}\right) .
$$

Since $X^{\prime}$ has independent coordinates of zero mean and unit variance, we get

$$
\begin{aligned}
\mathbb{E}\left(\left\|\mathrm{P}_{0} X^{\prime}\right\|^{4}\right) & \leq 8 \mathbb{E}\left(\left\|\mathrm{P} X^{\prime}\right\|^{4}\right)+8 \mathbb{E}\left(\left\|\left(\mathrm{P}-\mathrm{P}_{0}\right) X^{\prime}\right\|^{4}\right) \\
& \leq 8 \mathbb{E}\left(\sum_{i, j} \mathrm{P}_{i j} \xi_{i} \xi_{j}\right)^{2}+8 \mathbb{E}\left(\sum_{i=1}^{n} \xi_{i}^{2} \mathrm{P}_{i i}{ }^{2}\right)^{2} \\
& \leq 8 \max (B, 2) \sum_{i, j} \mathrm{P}_{i j}{ }^{2}+8 \sum_{i \neq j} \mathrm{P}_{i i} \mathrm{P}_{j j}+8 B \sum_{i, j} \mathrm{P}_{i i}{ }^{2} \mathrm{P}_{j j}{ }^{2} \\
& \leq 8 \max (B, 2) r+8 r^{2}+8 B r^{2} \\
& \leq 32 B r^{2} .
\end{aligned}
$$

Hence, $\mathbb{E}\left(\left\langle X, \mathrm{P}_{0} X\right\rangle^{4}\right) \leq(256 B r)^{2}$, and applying Markov's inequality, we get the following bound for $(*)$ :

$$
(*) \leq \frac{(1024 B r)^{2}}{u^{4}} .
$$

Let us turn to estimating $(* *)$. We will use symmetrization, truncation, and concentration. Let $\widetilde{X}=\left(\widetilde{\xi}_{k}\right)_{1 \leq k \leq n}$ be an independent copy of $X$. Note that

$$
\mathbb{E}\left(\left(\left\langle\widetilde{X},\left(\mathrm{P}-\mathrm{P}_{0}\right) \widetilde{X}\right\rangle-r\right)^{2}\right)=\mathbb{E}\left(\left(\sum_{k=1}^{n} \mathrm{P}_{k k}\left(\widetilde{\xi}_{k}^{2}-1\right)\right)^{2}\right) \leq B r,
$$

so, using the independence of $X$ and $\widetilde{X}$ and applying Markov's inequality, we get

$$
\begin{aligned}
(* *) & \leq 2 \mathbb{P}\left(\left|\left\langle X,\left(\mathrm{P}-\mathrm{P}_{0}\right) X\right\rangle-\left\langle\widetilde{X},\left(\mathrm{P}-\mathrm{P}_{0}\right) \tilde{X}\right\rangle\right|>u / 2-\sqrt{2 B r}\right) \\
& =2 \mathbb{P}\left(\left|\sum_{k=1}^{n} \mathrm{P}_{k k}\left(\xi_{k}^{2}-\widetilde{\xi}_{k}^{2}\right)\right|>u / 2-\sqrt{2 B r}\right) .
\end{aligned}
$$

Clearly, the variables $\xi_{k}^{2}-\widetilde{\xi}_{k}^{2}(1 \leq k \leq n)$ are symmetrically distributed, with the variance bounded from above by $2 B$. Let $h: \mathbb{R}_{+} \rightarrow \mathbb{R}_{+}$be a function defined as

$$
h(t):=\mathbb{E}\left(\left(\xi_{k}^{2}-\widetilde{\xi}_{k}^{2}\right)^{2} \chi_{t}\right),
$$

where $\chi_{t}$ denotes the indicator of the event $\left\{\left|\xi_{k}^{2}-\widetilde{\xi}_{k}^{2}\right| \geq t\right\}$. Clearly, $h(t) \rightarrow 0$ when $t$ tends to infinity. Note that, by Hoeffding's inequality, we have

$$
\begin{aligned}
\mathbb{P}\left(\left|\sum_{k=1}^{n} \mathrm{P}_{k k}\left(\xi_{k}^{2}-\widetilde{\xi}_{k}^{2}\right)\left(1-\chi_{r^{1 / 4}}\right)\right|>u / 4\right) & \leq 2 \exp \left(-\frac{2 u^{2}}{16 \sqrt{r}}\left(4 \sum_{k} \mathrm{P}_{k k}^{2}\right)^{-1}\right) \\
& \leq 2 \exp \left(-u^{2} /(32 r \sqrt{r})\right) .
\end{aligned}
$$

On the other hand, since the random variable $\left(\xi_{k}^{2}-\widetilde{\xi}_{k}^{2}\right) \chi_{r^{1 / 4}}$ has zero mean, we have

$$
\mathbb{E}\left(\left(\sum_{k=1}^{n} \mathrm{P}_{k k}\left(\xi_{k}^{2}-\widetilde{\xi}_{k}^{2}\right) \chi_{r^{1 / 4}}\right)^{2}\right) \leq r h\left(r^{1 / 4}\right) .
$$

Applying Markov's inequality, we get for all $u>4 \sqrt{2 B r}$,

$$
\mathbb{P}\left(\left|\sum_{k=1}^{n} \mathrm{P}_{k k}\left(\xi_{k}^{2}-\widetilde{\xi}_{k}^{2}\right) \chi_{r^{1 / 4}}\right|>u / 4-\sqrt{2 B r}\right) \leq \frac{r h\left(r^{1 / 4}\right)}{(u / 4-\sqrt{2 B r})^{2}} .
$$


Combining the estimates, we obtain

$$
\begin{aligned}
(* *) \leq & 2 \mathbb{P}\left(\left|\sum_{k=1}^{n} \mathrm{P}_{k k}\left(\xi_{k}^{2}-\widetilde{\xi}_{k}^{2}\right)\left(1-\chi_{r^{1 / 4}}\right)\right|>u / 4\right) \\
& +2 \mathbb{P}\left(\left|\sum_{k=1}^{n} \mathrm{P}_{k k}\left(\xi_{k}^{2}-\widetilde{\xi}_{k}^{2}\right) \chi_{r^{1 / 4}}\right|>u / 4-\sqrt{2 B r}\right) \\
\leq & 4 \exp \left(-u^{2} /(32 r \sqrt{r})\right)+\frac{128 r h\left(r^{1 / 4}\right)}{u^{2}} \text { for all } u>8 \sqrt{2 B r} .
\end{aligned}
$$

Finally, grouping together the bounds for $(*)$ and $(* *)$, we get for all $u>8 \sqrt{2 B r}$,

$$
\mathbb{P}\left(\left|\|\mathrm{P} X\|^{2}-r\right|>u\right) \leq \frac{(1024 B r)^{2}}{u^{4}}+4 \exp \left(-u^{2} /(32 r \sqrt{r})\right)+\frac{128 r h\left(r^{1 / 4}\right)}{u^{2}} .
$$

Set $\alpha \in(0,1 / 6)$. For any $u>8 \sqrt{2 B} r^{1-\alpha}=o(r)$, using the inequality $e^{-2 t} \leq 1 / t^{4}$ for $t>0$, the right hand side of the last equation above is bounded above by

$$
\frac{r}{u^{2}} \frac{(1024 B)^{2} r}{u^{2}}+\frac{r}{u^{2}} 4 \frac{64^{4} r^{5}}{u^{6}}+\frac{r}{u^{2}} 128 h\left(r^{1 / 4}\right)=\frac{o(r)}{u^{2}} .
$$

This proves the desired result. We note that the proof can be shortened and simplified under the stronger assumption that $\mathbb{E}\left(\left|\xi_{1}\right|^{p}\right)<\infty$ for some $p>4$, see also [SV13, Proposition 1.3] for thin-shell estimates in the same spirit.

\section{Proof of Theorem 1.5}

As in BSS12, SV13], for every $t \in \mathbb{R} \backslash\left\{\lambda_{1}(S), \ldots, \lambda_{n}(S)\right\}$, we set

$$
\underline{m}_{S}(t):=\operatorname{tr}\left(\left(S-t I_{n}\right)^{-1}\right)=\sum_{k=1}^{n} \frac{1}{\lambda_{k}(S)-t}
$$

The function $t \mapsto \underline{m}_{S}(t)$ is positive and strictly increasing on $\left(-\infty, \lambda_{n}(S)\right)$. We note that $n \underline{m}_{S}$ is the restriction on $\mathbb{R}$ of the Cauchy-Stieltjes transform of the empirical spectral distribution of $S$. What is important to us is that $\underline{m}_{S}$ encodes as singularities the eigenvalues of $S$, is monotone on $\left(-\infty, \lambda_{n}(S)\right)$, and behaves nicely under rank one updates of $S$.

3.1. Feasible lower shift. Let $A$ be an $n \times n$ positive semi-definite non-random matrix with eigenvalues $\lambda_{\max }:=\lambda_{1} \geq \cdots \geq \lambda_{n}=: \lambda_{\min } \geq 0$ and a corresponding orthonormal basis of eigenvectors $\left(x_{i}\right)_{i=1}^{n}$, and let $u<\lambda_{\min }$. Further, assume that $x$ is a (non-random) vector in $\mathbb{R}^{n}$. We are interested in those numbers $\delta \geq 0$ that (deterministically) satisfy

$$
\lambda_{\min }>u+\delta \quad \text { and } \quad \underline{m}_{A+x x^{\top}}(u+\delta) \leq \underline{m}_{A}(u) .
$$

Following [SV13, any value of $\delta$ satisfying (3.1), will be called a feasible lower shift with respect to $A, x$ and $u$. The following statement is taken from [SV13]; we provide its proof for reader's convenience.

Lemma 3.1 (Feasible lower shift - [SV13, Lemma 2.2]). Let $\delta \geq 0$ be such that $u+\delta<$ $\lambda_{\min }$. Let us define

$$
\begin{aligned}
& q_{1}(\delta):=x^{\top}(A-u-\delta)^{-1} x=\sum_{i=1}^{n} \frac{\left\langle x, x_{i}\right\rangle^{2}}{\lambda_{i}-u-\delta} \\
& q_{2}(\delta):=\frac{x^{\top}(A-u-\delta)^{-2} x}{\operatorname{tr}\left((A-u-\delta)^{-2}\right)}=\left(\sum_{i=1}^{n}\left(\lambda_{i}-u-\delta\right)^{-2}\right)^{-1} \sum_{i=1}^{n} \frac{\left\langle x, x_{i}\right\rangle^{2}}{\left(\lambda_{i}-u-\delta\right)^{2}} .
\end{aligned}
$$

Then a sufficient condition for (3.1) to be satisfied is $q_{2}(\delta) \geq \delta\left(1+q_{1}(\delta)\right)$. 
Proof. If $S$ is a symmetric matrix and if $x$ is a vector, and if both $S$ and $S+x x^{\top}$ are invertible, then $1+x^{\top} S^{-1} x \neq 0$ since $x^{\top} S^{-1} x=-1$ gives $\left(S+x x^{\top}\right) S^{-1} x=0$. Moreover the inverse $\left(S+x x^{\top}\right)^{-1}$ of the rank one update $S+x x^{\top}$ of $S$ can be expressed as

$$
\left(S+x x^{\top}\right)^{-1}=S^{-1}-\frac{S^{-1} x x^{\top} S^{-1}}{1+x^{\top} S^{-1} x} .
$$

This is known as the Sherman-Morrison formula. This allows to write

$$
\begin{aligned}
\underline{m}_{A+x x^{\top}}(u+\delta) & =\operatorname{tr}\left(\left(A-u-\delta+x x^{\top}\right)^{-1}\right) \\
& =\operatorname{tr}\left((A-u-\delta)^{-1}\right)-\frac{x^{\top}(A-u-\delta)^{-2} x}{1+x^{\top}(A-u-\delta)^{-1} x} \\
& =\underline{m}_{A}(u)+\operatorname{tr}\left((A-u-\delta)^{-1}-(A-u)^{-1}\right)-\frac{x^{\top}(A-u-\delta)^{-2} x}{1+x^{\top}(A-u-\delta)^{-1} x} .
\end{aligned}
$$

Now since $A-(u+\delta) I_{n}$ is positive definite, it follows that

$$
\delta(A-u-\delta)^{-2}-\left((A-u-\delta)^{-1}-(A-u)^{-1}\right)
$$

is positive definite, and therefore

$$
\underline{m}_{A+x x^{\top}}(u+\delta)-\underline{m}_{A}(u) \leq \delta \operatorname{tr}\left((A-u-\delta)^{-2}\right)-\frac{x^{\top}(A-u-\delta)^{-2} x}{1+x^{\top}(A-u-\delta)^{-1} x} .
$$

Finally it can be checked that the right hand side is $\leq 0$ if $\delta\left(1+q_{1}(\delta)\right)-q_{2}(\delta) \leq 0$.

Lemma 3.2 (Construction of the feasible shift). Let $A, x, u$ and $q_{1}, q_{2}$ be as above, $\varepsilon \in(0,1)$ and assume that

$$
\lambda_{\min }-u \geq 2 / \varepsilon^{2}
$$

Then the quantity

$$
\delta:=\frac{(1-\varepsilon) \mathbf{1}_{\left\{q_{1}(1 / \varepsilon) \leq(1+\varepsilon) \underline{m}_{A}(u)+\varepsilon\right\}}}{(1+\varepsilon)\left(1+\underline{m}_{A}(u)\right)}\left(\sum_{i=1}^{n}\left(\lambda_{i}-u\right)^{-2}\right)^{-1} \sum_{i=1}^{n} \frac{\left\langle x, x_{i}\right\rangle^{2} \mathbf{1}_{\left\{\left\langle x, x_{i}\right\rangle^{2} \leq 1 / \varepsilon\right\}}}{\left(\lambda_{i}-u\right)^{2}}
$$

satisfies $\delta \leq 1 / \varepsilon$ and is a feasible lower shift w.r.t. $A, x$ and $u$, i.e. $\lambda_{\min }>u+\delta$ and $q_{2}(\delta) \geq \delta\left(1+q_{1}(\delta)\right)$.

Proof. First, note that the condition $\lambda_{\min }-u \geq 2 / \varepsilon^{2}$ immediately implies that

$$
\varepsilon\left(\lambda_{i}-u\right)^{2}-\frac{2\left(\lambda_{i}-u\right)}{\varepsilon}+\frac{1}{\varepsilon^{2}} \geq 0, \quad i=1, \ldots, n,
$$

which is in turn equivalent to the relation

$$
\frac{1}{(1-\varepsilon)\left(\lambda_{i}-u\right)^{2}} \geq \frac{1}{\left(\lambda_{i}-u-1 / \varepsilon\right)^{2}}, \quad i=1, \ldots, n .
$$

Now, let us return to $\delta$. The inequality $\delta \leq 1 / \varepsilon$ follows directly from its definition. Next,

$$
\frac{\left(1+q_{1}(\delta)\right) \mathbf{1}_{\left\{q_{1}(1 / \varepsilon) \leq(1+\varepsilon) \underline{m}_{A}(u)+\varepsilon\right\}}}{(1+\varepsilon)\left(1+\underline{m}_{A}(u)\right)} \leq 1,
$$

so we get

$$
\delta\left(1+q_{1}(\delta)\right) \leq(1-\varepsilon) q_{2}(0) \leq q_{2}(\delta),
$$

where the last inequality comes from (3.3) and the definition of $q_{2}$. 
3.2. Randomization and control of expectations. Let, as before, $A$ be an $n \times n$ nonrandom positive semidefinite matrix with eigenvalues $\lambda_{1} \geq \cdots \geq \lambda_{n} \geq 0$, and let $u<\lambda_{n}$. We define a (random) quantity $\delta$ as in Lemma 3.2, replacing the fixed vector $x$ with a random isotropic vector $X$.

Lemma 3.3. Let $\varepsilon \in\left(0,12^{-3}\right), n \geq 12 / \varepsilon^{4}$, and let $X$ be a random isotropic vector in $\mathbb{R}^{n}$ such that

$$
\mathbb{P}\left(\|\mathrm{P} X\|^{2}-\operatorname{rankP} \geq \varepsilon \operatorname{rank} \mathrm{P} / 6\right) \leq \varepsilon^{4}
$$

for any non-zero orthogonal projection $\mathrm{P}$ of rank at least $\varepsilon^{11} n / 72$. Further, let the nonrandom matrix $A$, the numbers $\left(\lambda_{i}\right)_{i \leq n}$ and vectors $\left(x_{i}\right)_{i \leq n}$ be as above, and $u \in \mathbb{R}$ be such that $\lambda_{\min }-u \geq 6 \varepsilon^{-2}+\varepsilon^{-1}$. Assume additionally that

$$
\sum_{i=1}^{n} \frac{1}{\left(\lambda_{i}-u\right)\left(\lambda_{i}-u+1\right)} \leq \frac{1}{\varepsilon n} .
$$

Then, with $q_{1}$ defined as in Lemma 3.1] (with $X$ replacing the non-random vector $x$ ), we have

$$
\mathbb{P}\left\{q_{1}(1 / \varepsilon)+1 \geq(1+\varepsilon)\left(1+\underline{m}_{A}(u)\right)\right\} \leq 4 \varepsilon^{2} .
$$

Proof. First, note that the lower bound on $\lambda_{\min }-u$ implies that $q_{1}(1 / \varepsilon) \leq(1+\varepsilon / 6) q_{1}(0)$ (deterministically). Let us split the index set $\{1, \ldots, n\}$ into several subsets in the following way: First, let $I:=\left\{i \leq n: \lambda_{i}-u \leq \varepsilon^{4} n / 12\right\}$. Next, we set

$$
J:=\left\{i \leq n: \lambda_{i}-u \geq 6 n / \varepsilon^{3}\right\},
$$

so that

$$
\{1, \ldots, n\} \backslash(I \cup J)=\left\{i \leq n: \varepsilon^{4} n / 12<\lambda_{i}-u<6 n / \varepsilon^{3}\right\} .
$$

Note that, by the choice of $\varepsilon$, we have $\exp (1 /(12 \varepsilon)) \geq 72 / \varepsilon^{7}$. Hence, the interval $\left(\ln \left(\varepsilon^{4} n / 12\right), \ln \left(6 n / \varepsilon^{3}\right)\right)$ can be partitioned into $\left\lfloor\varepsilon^{-2}\right\rfloor$ subintervals $\mathcal{S}_{k}\left(k \leq \varepsilon^{-2}\right)$ of length at most $\varepsilon / 6$ each. Then we let

$$
I_{k}:=\left\{i \leq n: \ln \left(\lambda_{i}-u\right) \in \mathcal{S}_{k}\right\}, \quad k \leq \varepsilon^{-2} .
$$

Obviously,

$$
q_{1}(0)=\sum_{i \in I} \frac{\left\langle X, x_{i}\right\rangle^{2}}{\lambda_{i}-u}+\sum_{i \in J} \frac{\left\langle X, x_{i}\right\rangle^{2}}{\lambda_{i}-u}+\sum_{k \leq \varepsilon^{-2}} \sum_{i \in I_{k}} \frac{\left\langle X, x_{i}\right\rangle^{2}}{\lambda_{i}-u}=(*)+(* *)+(* * *) .
$$

Let us estimate the three quantities separately.

First, in view of the condition (3.4), the lower bound on $n$ and the definition of $I$, we have

$$
\mathbb{E}(*)=\sum_{i \in I} \frac{1}{\lambda_{i}-u} \leq \sum_{i \in I} \frac{\varepsilon^{4} n / 12+1}{\left(\lambda_{i}-u\right)\left(\lambda_{i}-u+1\right)} \leq \frac{\varepsilon^{3}}{6} .
$$

Hence, by Markov's inequality,

$$
\mathbb{P}\{(*) \geq \varepsilon / 6\} \leq \varepsilon^{2} .
$$

Similarly,

whence

$$
\mathbb{E}(* *)=\sum_{i \in J} \frac{1}{\lambda_{i}-u} \leq \frac{\varepsilon^{3}}{6},
$$

$$
\mathbb{P}\{(* *) \geq \varepsilon / 6\} \leq \varepsilon^{2} .
$$

Now, we consider the quantity $(* * *)$. First, assume that for some $k \leq \varepsilon^{-2}$ we have $\left|I_{k}\right| \leq \varepsilon^{11} n / 72$. Since for every $i \in I_{k}$ we have $\lambda_{i}-u \geq \varepsilon^{4} n / 12$, we obtain

$$
\mathbb{E} \sum_{i \in I_{k}} \frac{\left\langle X, x_{i}\right\rangle^{2}}{\lambda_{i}-u} \leq \frac{\varepsilon^{7}}{6}
$$


whence, by Markov's inequality,

$$
\mathbb{P}\left\{\sum_{i \in I_{k}} \frac{\left\langle X, x_{i}\right\rangle^{2}}{\lambda_{i}-u} \geq \varepsilon^{3} / 6\right\} \leq \varepsilon^{4} .
$$

Now, if for some $k \leq \varepsilon^{-2}$ we have $\left|I_{k}\right|>\varepsilon^{11} n / 72$, then, by the condition on projections and the definition of $I_{k}$, denoting by $P_{k}$ the orthogonal projection onto the span of $\left(x_{i}\right)_{i \in I_{k}}$, we obtain

$$
\mathbb{P}\left\{\sum_{i \in I_{k}} \frac{\left\langle X, x_{i}\right\rangle^{2}}{\lambda_{i}-u} \geq \sum_{i \in I_{k}} \frac{\exp (\varepsilon / 6)(1+\varepsilon / 6)}{\lambda_{i}-u}\right\} \leq \mathbb{P}\left\{\left\|\mathrm{P}_{k} X\right\|^{2} \geq(1+\varepsilon / 6) \operatorname{rankP}_{k}\right\} \leq \varepsilon^{4} .
$$

Combining all the above estimates together, we get

$$
\begin{aligned}
& \mathbb{P}\left\{q_{1}(1 / \varepsilon) \geq(1+\varepsilon / 6)\left(\varepsilon / 2+\exp (\varepsilon / 6)(1+\varepsilon / 6) \underline{m}_{A}(u)\right)\right\} \\
& \quad \leq \mathbb{P}\left\{(*)+(* *)+(* * *) \geq \varepsilon / 6+\varepsilon / 6+\varepsilon / 6+\exp (\varepsilon / 6)(1+\varepsilon / 6) \underline{m}_{A}(u)\right\} \\
& \quad \leq 4 \varepsilon^{2} .
\end{aligned}
$$

It remains to note that

$$
(1+\varepsilon / 6)\left(\varepsilon / 2+\exp (\varepsilon / 6)(1+\varepsilon / 6) \underline{m}_{A}(u)\right)+1 \leq(1+\varepsilon)\left(1+\underline{m}_{A}(u)\right) .
$$

Lemma 3.4 (Control of $\mathbb{E} \delta$ ). Let $\varepsilon \in\left(0,12^{-3}\right), n \geq 12 / \varepsilon^{4}$, and let $A,\left(\lambda_{i}\right)_{i \leq n},\left(x_{i}\right)_{i \leq n}, X$ and $\delta$ be the same as in Lemma 3.3 (and satisfy the same conditions). Assume additionally that $\mathbb{E}\left(\left\langle X, x_{i}\right\rangle^{2} \mathbf{1}_{\left\{\left\langle X, x_{i}\right\rangle^{2} \leq 1 / \varepsilon\right\}}\right) \geq r(i \leq n)$ for some $r \in[0,1]$. Then

$$
\mathbb{E} \delta \geq \frac{(1-\varepsilon) r}{(1+\varepsilon)\left(1+\underline{m}_{A}(u)\right)}-4 \varepsilon
$$

Proof. Since

$$
\delta=\frac{(1-\varepsilon)\left(1-\mathbf{1}_{\left\{q_{1}(1 / \varepsilon)>(1+\varepsilon) \underline{m}_{A}(u)+\varepsilon\right\}}\right)}{(1+\varepsilon)\left(1+\underline{m}_{A}(u)\right)}\left(\sum_{i=1}^{n}\left(\lambda_{i}-u\right)^{-2}\right)^{-1} \sum_{i=1}^{n} \frac{\left\langle X, x_{i}\right\rangle^{2} \mathbf{1}_{\left\{\left\langle X, x_{i}\right\rangle^{2} \leq 1 / \varepsilon\right\}}}{\left(\lambda_{i}-u\right)^{2}},
$$

and in view of the bound $\delta \leq 1 / \varepsilon$, we get

$$
\begin{aligned}
\mathbb{E} \delta & \geq \frac{(1-\varepsilon)}{(1+\varepsilon)\left(1+\underline{m}_{A}(u)\right)}\left(\sum_{i=1}^{n}\left(\lambda_{i}-u\right)^{-2}\right)^{-1} \sum_{i=1}^{n} \frac{\mathbb{E}\left(\left\langle X, x_{i}\right\rangle^{2} \mathbf{1}_{\left\{\left\langle X, x_{i}\right\rangle^{2} \leq 1 / \varepsilon\right\}}\right)}{\left(\lambda_{i}-u\right)^{2}} \\
& -\varepsilon^{-1} \mathbb{P}\left\{q_{1}(1 / \varepsilon)>(1+\varepsilon) \underline{m}_{A}(u)+\varepsilon\right\} .
\end{aligned}
$$

Finally, applying Lemma 3.3 to the last expression, we get the result.

3.3. Proof of Theorem 1.5, completed. Let $\left(X_{n}\right)_{n \in \mathbb{N}}$ be as in the statement of the theorem. Without loss of generality, we can assume that both functions $f, g: \mathbb{N} \rightarrow \mathbb{R}_{+}$in the Weak Tail Projection property (WTP-b) are non-increasing. Additionally let us define

$$
h(M):=\sup _{\substack{n \in \mathbb{N} \\ y \in S^{n-1}}} \mathbb{E}\left(\left\langle X_{n}, y\right\rangle^{2} \mathbf{1}_{\left\{\left\langle X_{n}, y\right\rangle^{2} \geq M\right\}}\right), \quad M \geq 0
$$

Note that (WTP-a gives $\lim _{M \rightarrow \infty} h(M)=0$.

Take any $\varepsilon \in\left(0,12^{-3}\right)$ and define $n_{\varepsilon}$ as the smallest integer greater than $12 / \varepsilon^{4}$ such that (a) $g\left(\varepsilon^{11} n_{\varepsilon} / 72\right) \leq \varepsilon^{4}$ and $f\left(\varepsilon^{11} n_{\varepsilon} / 72\right) \leq \varepsilon / 6$ and (b) for all $n \geq n_{\varepsilon}$ we have $\left(\sqrt{m_{n}}-\sqrt{n}\right)^{2} \geq$ $\varepsilon$ and $m_{n} / n \geq 3 \bar{\rho}^{-1} / 4+1 / 4$ (the latter implies $\left(\sqrt{m_{n} / n}-1\right)^{-1} \leq 2 /\left(\bar{\rho}^{-1 / 2}-1\right)$ ). From now on, we fix an $n \geq n_{\varepsilon}$, let $m:=m_{n}$ and let $X^{(1)}, \ldots, X^{(m)}$ be i.i.d. copies of $X_{n}$. We define

$$
A^{(0)}:=0 \quad \text { and } \quad A^{(k)}:=A^{(k-1)}+X^{(k)} \otimes X^{(k)}, \quad 1 \leq k \leq m,
$$

so that $A_{n}=A^{(m)}$. Set

$$
u_{0}:=n-\sqrt{m n}
$$


and let $u_{1}, \ldots, u_{m}$ be a collection of random numbers defined inductively as follows:

$$
u_{k}:=u_{k-1}+\delta^{k}-\delta_{R}^{k},
$$

where $\delta^{k}$ is defined as $\delta$ in Lemma 3.2 (with $A^{(k-1)}, u_{k-1}$ and $X^{(k)}$ replacing $A, u$ and $x$, respectively) and the regularity shift $\delta_{R}^{k}$ is defined by

$$
\delta_{R}^{k}:=\min \left\{\ell \in\{0,1, \ldots\}: \underline{m}_{A^{(k)}}\left(u_{k-1}+\delta^{k}-\ell\right)-\underline{m}_{A^{(k)}}\left(u_{k-1}+\delta^{k}-\ell-1\right) \leq \frac{1}{\varepsilon n}\right\} .
$$

Note that lemmas 3.1 and 3.2 imply that we have, (deterministically) for all $k \geq 0$,

$$
\underline{m}_{A^{(k)}}\left(u_{k}\right) \leq \underline{m}_{A^{(0)}}\left(u_{0}\right)=\frac{n}{\sqrt{m n}-n} .
$$

The ultimate purpose of the shift $\delta_{R}^{k}$ is to guarantee relation (3.4) which was an important condition in proving the concentration lemma 3.3. Of course, since $\delta_{R}^{k}$ moves $u_{k}$ away from the spectrum, one must make sure that the cumulative impact of the shifts $\delta_{R}^{k}$ is small enough and does not destroy the desired asymptotic estimate.

Lemma 3.5. With $\delta_{R}^{k}(1 \leq k \leq m)$ defined above, the following holds deterministically:

$$
\sum_{k=1}^{m} \delta_{R}^{k} \leq \frac{\varepsilon n^{2}}{\sqrt{m n}-n} .
$$

Proof. Take any admissible $k \geq 1$. The definition of $\delta_{R}^{k}$ immediately implies that for all $0 \leq \ell<\delta_{R}^{k}$ we have

$$
\underline{m}_{A^{(k)}}\left(u_{k-1}+\delta^{k}-\ell\right)-\underline{m}_{A^{(k)}}\left(u_{k-1}+\delta^{k}-\ell-1\right)>\frac{1}{\varepsilon n} .
$$

Hence,

$$
\underline{m}_{A^{(k)}}\left(u_{k}\right)=\underline{m}_{A^{(k)}}\left(u_{k-1}+\delta^{k}-\delta_{R}^{k}\right) \leq \underline{m}_{A^{(k)}}\left(u_{k-1}+\delta^{k}\right)-\frac{\delta_{R}^{k}}{\varepsilon n} \leq \underline{m}_{A^{(k-1)}}\left(u_{k-1}\right)-\frac{\delta_{R}^{k}}{\varepsilon n} .
$$

Thus, $\underline{m}_{A^{(k)}}\left(u_{k}\right) \leq \underline{m}_{A^{(k-1)}}\left(u_{k-1}\right)-\frac{\delta_{R}^{k}}{\varepsilon n}$ for all $k \geq 1$, which, together with the relations $0<\underline{m}_{A^{(m)}}\left(u_{m}\right)$ and $\underline{m}_{A^{(0)}}\left(u_{0}\right)=\frac{n}{\sqrt{m n}-n}$ implies the result.

Now, fix for a moment any $k \in\{1, \ldots, m\}$ and let $\mathcal{F}_{k-1}$ be the $\sigma$-algebra generated by the vectors $X^{(1)}, \ldots, X^{(k-1)}$, with the convention $\mathcal{F}_{0}=\{\varnothing, \Omega\}$. We will first estimate the conditional expectation $\mathbb{E}\left(\delta^{k} \mid \mathcal{F}_{k-1}\right)$.

Note that, by the definition of $u_{k-1}$, we have (deterministically)

$$
\underline{m}_{A^{(k-1)}}\left(u_{k-1}\right)-\underline{m}_{A^{(k-1)}}\left(u_{k-1}-1\right) \leq \frac{1}{\varepsilon n}
$$

(the above relation holds for $k>1$ in view of the definition of $\delta_{R}^{k-1}$ and for $k=1$ because of the definition of $n_{\varepsilon}$ ). Together with the lower bound on $n$ (which implies the conditions on orthogonal projections assumed in Lemma 3.4) and the condition

$$
\underline{m}_{A^{(k-1)}}\left(u_{k-1}\right) \leq \underline{m}_{A^{(0)}}\left(u_{0}\right)=\frac{n}{\sqrt{m n}-n},
$$

we get from Lemma 3.4 that

$$
\mathbb{E}\left(\delta^{k} \mid \mathcal{F}_{k-1}\right) \geq \frac{(1-\varepsilon)(1-h(1 / \varepsilon))}{(1+\varepsilon)\left(1+\underline{m}_{A^{(0)}}\left(u_{0}\right)\right)}-4 \varepsilon=\frac{(1-\varepsilon)(1-h(1 / \varepsilon))}{1+\varepsilon}\left(1-\sqrt{\frac{n}{m}}\right)-4 \varepsilon .
$$

Hence, by the definition of $u_{k}$ 's and Lemma 3.5, we obtain

$$
\mathbb{E} u_{m} \geq u_{0}+\frac{(1-\varepsilon)(1-h(1 / \varepsilon))}{1+\varepsilon}(m-\sqrt{m n})-4 \varepsilon m-\frac{\varepsilon n^{2}}{\sqrt{m n}-n} .
$$


Since $u_{m}<\lambda_{\min }\left(A_{n}\right)$ (deterministically), we get from the above relation

$$
\begin{aligned}
\mathbb{E} \lambda_{\min }\left(A_{n}\right) & \geq(\sqrt{m}-\sqrt{n})^{2}-(3 \varepsilon+h(1 / \varepsilon))(m-\sqrt{m n})-4 \varepsilon m-\frac{\varepsilon n^{2}}{\sqrt{m n}-n} \\
& \geq(\sqrt{m}-\sqrt{n})^{2}-7 \varepsilon m-h(1 / \varepsilon) m-\frac{2 \varepsilon n}{\bar{\rho}^{-1 / 2}-1} .
\end{aligned}
$$

Since the above estimate holds for arbitrarily small $\varepsilon$, and having in mind that $h(1 / \varepsilon) \rightarrow$ 0 with $\varepsilon \rightarrow 0$, we get the desired result.

\section{Proof of Theorem 1.7}

Following [BSS12, SV13, for arbitrary $n \times n$ symmetric matrix $S$ and every $t \in \mathbb{R} \backslash$ $\left\{\lambda_{1}(S), \ldots, \lambda_{n}(S)\right\}$, we set

$$
\bar{m}_{S}(t):=\operatorname{tr}\left(\left(t I_{n}-S\right)^{-1}\right)=\sum_{k=1}^{n} \frac{1}{t-\lambda_{k}(S)} .
$$

The function $t \mapsto \bar{m}_{S}(t)=-\underline{m}_{S}(t)$ is positive and strictly decreasing on $\left(\lambda_{1}(S),+\infty\right)$.

4.1. Feasible upper shift. Let $A$ be an $n \times n$ positive semi-definite non-random matrix with eigenvalues $\lambda_{\max }:=\lambda_{1} \geq \cdots \geq \lambda_{n}=: \lambda_{\min } \geq 0$ and a corresponding orthonormal basis of eigenvectors $\left(x_{i}\right)_{i=1}^{n}$, and let $u>\lambda_{1}$ be such that $\bar{m}_{A}(u)<1$. Further, assume that $x$ is a (non-random) vector in $\mathbb{R}^{n}$. In this section, we consider those numbers $\Delta \geq 0$ that (deterministically) satisfy

$$
\lambda_{\max }\left(A+x x^{\top}\right)<u+\Delta \quad \text { and } \quad \bar{m}_{A+x x^{\top}}(u+\Delta) \leq \bar{m}_{A}(u) .
$$

Following [SV13], any value of $\Delta$ satisfying (4.1), will be called a feasible upper shift with respect to $A, x$ and $u$. The following statement is taken from [SV13]; we provide its proof for completeness.

Lemma 4.1 (Feasible upper shift - [SV13, Lemma 3.3]). Let $u>\lambda_{1}$ and $\bar{m}_{A}(u)<1$. For any $\Delta>0$, define

$$
\begin{aligned}
Q_{1}(\Delta) & :=x^{\top}(u+\Delta-A)^{-1} x=\sum_{i=1}^{n} \frac{\left\langle x, x_{i}\right\rangle^{2}}{u+\Delta-\lambda_{i}} \\
Q_{2}(\Delta) & :=\frac{x^{\top}(u+\Delta-A)^{-2} x}{\bar{m}_{A}(u)-\bar{m}_{A}(u+\Delta)}=\left(\bar{m}_{A}(u)-\bar{m}_{A}(u+\Delta)\right)^{-1} \sum_{i=1}^{n} \frac{\left\langle x, x_{i}\right\rangle^{2}}{\left(u+\Delta-\lambda_{i}\right)^{2}} .
\end{aligned}
$$

Then a sufficient condition for (4.1) to be satisfied is

$$
Q_{1}(\Delta)<1 \text { and } Q_{2}(\Delta) \leq 1-Q_{1}(\Delta)
$$

Proof. We can assume without loss of generality that $x \neq 0$, so that $Q_{2}(\Delta)>0$. The Sherman-Morrison formula (3.2) gives

$$
\begin{aligned}
\bar{m}_{A+x x^{\top}}(u+\Delta) & =\operatorname{tr}\left((u+\Delta) I_{n}-A-x x^{\top}\right)^{-1} \\
& =\operatorname{tr}\left((u+\Delta) I_{n}-A\right)^{-1}+\frac{\left\|\left((u+\Delta) I_{n}-A\right)^{-1} x\right\|^{2}}{1-x^{\top}\left((u+\Delta) I_{n}-A\right)^{-1} x} \\
& =\bar{m}_{A}(u+\Delta)+\left(1-\sum_{i=1}^{n} \frac{\left\langle x, x_{i}\right\rangle^{2}}{u+\Delta-\lambda_{i}}\right)^{-1} \sum_{i=1}^{n} \frac{\left\langle x, x_{i}\right\rangle^{2}}{\left(u+\Delta-\lambda_{i}\right)^{2}} .
\end{aligned}
$$

Hence, we have $\bar{m}_{A+x x^{\top}}(u+\Delta) \leq \bar{m}_{A}(u)$ if and only if

$$
\left(1-\sum_{i=1}^{n} \frac{\left\langle x, x_{i}\right\rangle^{2}}{u+\Delta-\lambda_{i}}\right)^{-1} \sum_{i=1}^{n} \frac{\left\langle x, x_{i}\right\rangle^{2}}{\left(u+\Delta-\lambda_{i}\right)^{2}} \leq \bar{m}_{A}(u)-\bar{m}_{A}(u+\Delta) .
$$

But the latter inequality clearly holds if $Q_{1}(\Delta)<1$ and $Q_{2}(\Delta) \leq 1-Q_{1}(\Delta)$. 
Next, the rank one matrix

$$
(u+\Delta-A)^{-1 / 2} x\left((u+\Delta-A)^{-1 / 2} x\right)^{\top}=(u+\Delta-A)^{-1 / 2} x x^{\top}(u+\Delta-A)^{-1 / 2}
$$

has eigenvalues 0 and $\left\|(u+\Delta-A)^{-1 / 2} x\right\|^{2}$. But the condition $Q_{1}(\Delta)<1$ implies $\|(u+$ $\Delta-A)^{-1 / 2} x \|^{2}=x^{\top}(u+\Delta-A)^{-1} x<1$. Therefore the matrix

$$
I_{n}-(u+\Delta-A)^{-1 / 2} x x^{\top}(u+\Delta-A)^{-1 / 2}
$$

is positive definite, implying that

$$
(u+\Delta-A)^{1 / 2} I_{n}(u+\Delta-A)^{1 / 2}-x x^{\top}=(u+\Delta) I_{n}-\left(A+x x^{\top}\right)
$$

is positive definite (recall that for any two positive definite matrices $S$ and $T$, the matrix $S^{1 / 2} T S^{1 / 2}$ is positive definite). Hence, $u+\Delta>\lambda_{\max }\left(A+x x^{\top}\right)$.

Following [SV13, we will treat the quantities $Q_{1}$ and $Q_{2}$ separately: for a specially chosen number $\tau \in(0,1)$, we will take $\Delta_{1}$ such that $Q_{1}\left(\Delta_{1}\right) \leq \tau$, and $\Delta_{2}$ such that $Q_{2}\left(\Delta_{2}\right) \leq 1-\tau$. Then, in view of monotonicity of $Q_{1}$ and $Q_{2}$, the sum $\Delta_{1}+\Delta_{2}$ will be a feasible upper shift. In our case, $\tau$ shall be close to $\bar{m}_{A}(u)$.

Let us introduce "level sets" $I_{j}$ as follows:

$$
I_{j}:=\left\{i \leq n: 4^{j-1} \leq u-\lambda_{i}<4^{j}\right\}, \quad j \in \mathbb{N} .
$$

Note that the condition $\bar{m}_{A}(u)<1$ immediately implies that $\left|I_{j}\right|<4^{j}$ for all $j \geq 1$. Moreover, if we additionally assume that $\max _{\ell \in \mathbb{N}} \frac{\left|I_{\ell}\right|}{16^{\ell}}$ is sufficiently small then the following estimate of the ratio $\frac{\left|I_{j}\right|}{4^{j}}$ is valid:

Lemma 4.2. Let the sets $I_{j}$ be as above and assume that for some $\varepsilon \in(0,1]$ we have

$$
\max _{\ell \in \mathbb{N}} \frac{\left|I_{\ell}\right|}{16^{\ell}} \leq \frac{1}{\varepsilon n} .
$$

Then

$$
\frac{\left|I_{j}\right|}{4^{j}} \leq \sqrt{\frac{\left|I_{j}\right|}{\varepsilon n}}, \quad j \in \mathbb{N} .
$$

Proof. Fix any natural $j$ such that $I_{j} \neq \varnothing$. Then, obviously,

$$
\frac{\left|I_{j}\right|}{4^{j}} \leq \frac{4^{j}}{\varepsilon n}
$$

Fix for a moment any $\alpha>0$. If $\left|I_{j}\right| \geq \alpha 4^{j}$ then

$$
\frac{\left|I_{j}\right|}{4^{j}} \leq \frac{\alpha^{-1}\left|I_{j}\right|}{\varepsilon n} .
$$

Otherwise, $\frac{\left|I_{j}\right|}{4^{j}} \leq \alpha$. It remains to choose $\alpha:=\sqrt{\frac{\left|I_{j}\right|}{\varepsilon n}}$.

For every $j \geq 1$, denote

$$
h_{j}:=\sum_{i \in I_{j}}\left\langle x, x_{i}\right\rangle^{2}-\left|I_{j}\right| .
$$

Lemma 4.3 (Definition of $\Delta_{1}$ ). Let $A, x$ and $u$ be as above, with $u>\lambda_{\max }$ and $\bar{m}_{A}(u)<1$, and let $\varepsilon \in(0,1 / 4]$ be a real parameter. Define a number $\Delta_{1}$ as follows:

$$
\Delta_{1}:=\Delta_{1}^{\prime}+\sum_{j=1}^{\left\lfloor\log _{4}\left(n / \varepsilon^{2}\right)\right\rfloor} \Delta_{1, j},
$$

where

$$
\Delta_{1}^{\prime}:=\varepsilon^{-1 / 2}\|x\|^{2} \mathbf{1}_{\left\{\varepsilon\|x\|^{2} \geq n\right\}}
$$


and, for each natural $j \leq \log _{4}\left(n / \varepsilon^{2}\right)$, we let

$$
\Delta_{1, j}:=\varepsilon^{-1} h_{j} \mathbf{1}_{\left\{h_{j}>\varepsilon^{2} 2^{j} \sqrt{\left|I_{j}\right|}\right\}} .
$$

Let $Q_{1}$ be as in Lemma 4.1. Then $\Delta_{1}$ satisfies

$$
Q_{1}\left(\Delta_{1}\right) \leq \bar{m}_{A}\left(u+\Delta_{1}\right)+6 \sqrt{\varepsilon}+8 \varepsilon \sqrt{n} \sqrt{\max _{\ell \in \mathbb{N}} \frac{\left|I_{\ell}\right|}{16^{\ell}}} .
$$

Proof. Denote by $J$ the set of all $j \leq \log _{4}\left(n / \varepsilon^{2}\right)$ such that $h_{j}>\varepsilon^{2} 2^{j} \sqrt{\left|I_{j}\right|}$ and let $J^{\prime}$ be its complement inside $\left\{1, \ldots,\left\lfloor\log _{4}\left(n / \varepsilon^{2}\right)\right\rfloor\right\}$. Then

$$
\begin{aligned}
Q_{1}\left(\Delta_{1}\right) & =\bar{m}_{A}\left(u+\Delta_{1}\right)+\sum_{j=1}^{\infty} \sum_{i \in I_{j}} \frac{\left\langle x, x_{i}\right\rangle^{2}-1}{u+\Delta_{1}-\lambda_{i}} \\
& \leq \bar{m}_{A}\left(u+\Delta_{1}\right)+\sum_{j>\log _{4}\left(n / \varepsilon^{2}\right)} \sum_{i \in I_{j}} \frac{\left\langle x, x_{i}\right\rangle^{2}}{u+\Delta_{1}-\lambda_{i}}+4 \sum_{j \in J^{\prime}} \frac{h_{j}}{\Delta_{1}+4^{j}}+4 \sum_{j \in J} \frac{h_{j}}{\Delta_{1}+4^{j}} \\
& =: \bar{m}_{A}\left(u+\Delta_{1}\right)+(*)+4(* *)+4(* * *) .
\end{aligned}
$$

We shall estimate the last three quantities separately. First, note that

$$
(*) \leq \frac{4\|x\|^{2}}{\Delta_{1}+n / \varepsilon^{2}} \leq \frac{4 \sqrt{\varepsilon}\|x\|^{2}}{\|x\|^{2} \mathbf{1}_{\left\{\varepsilon\|x\|^{2} \geq n\right\}}+n / \varepsilon^{3 / 2}} \leq 4 \sqrt{\varepsilon} .
$$

Next,

$$
(* *) \leq \varepsilon^{2} \sum_{j \in J^{\prime}} \sqrt{\frac{\left|I_{j}\right|}{4^{j}}} \leq \varepsilon^{2} \sqrt{\max _{\ell \in \mathbb{N}} \frac{\left|I_{\ell}\right|}{16^{\ell}}} \sum_{j \leq \log _{4}\left(n / \varepsilon^{2}\right)} 2^{j} \leq 2 \varepsilon \sqrt{n} \sqrt{\max _{\ell \in \mathbb{N}} \frac{\left|I_{\ell}\right|}{16^{\ell}}} .
$$

Finally, we have

$$
(* * *) \leq \frac{\sum_{j \in J} h_{j}}{\sum_{j \in J} \Delta_{1, j}} \leq \varepsilon .
$$

Summing up the estimates, we get the result.

Denote

$$
F_{2}(\Delta):=\left(\sum_{i=1}^{n} \frac{1}{\left(u+\Delta-\lambda_{i}\right)\left(u-\lambda_{i}\right)}\right)^{-1} \sum_{i=1}^{n} \frac{\left\langle x, x_{i}\right\rangle^{2}}{\left(u+\Delta-\lambda_{i}\right)^{2}}, \quad \Delta \geq 0
$$

(clearly, $F_{2}(\Delta)=\Delta Q_{2}(\Delta)$ for all $\Delta>0$ ).

Lemma 4.4 (Definition of $\Delta_{2}$ ). Let $A$, $u$ and $x$ be as above, with $u>\lambda_{1}$, and let $\alpha>0$ satisfy $\bar{m}_{A}(u)+\alpha<1$. Define $\Delta_{2}$ according to the following procedure: if

$$
(1+\alpha) F_{2}(0) \leq \alpha\left(u-\lambda_{1}\right)\left(1-\bar{m}_{A}(u)-\alpha\right)
$$

then set

$$
\Delta_{2}:=\frac{(1+\alpha) F_{2}(0)}{1-\bar{m}_{A}(u)-\alpha},
$$

otherwise, take the smallest non-negative integer $j$ such that

$$
Q_{2}\left(2^{j}\left(u-\lambda_{1}\right)\right) \leq 1-\bar{m}_{A}(u)-\alpha
$$

and let

$$
\Delta_{2}:=2^{j}\left(u-\lambda_{1}\right) .
$$

Then $\Delta_{2}=0$ whenever $x=0$, and, for $x \neq 0$, we have $\Delta_{2} \neq 0$ and

$$
Q_{2}\left(\Delta_{2}\right) \leq 1-\bar{m}_{A}(u)-\alpha .
$$


Proof. The case $x=0$ is trivial, so further we assume that $x \neq 0$ implying $\Delta_{2} \neq 0$. First, suppose that

$$
(1+\alpha) F_{2}(0) \leq \alpha\left(u-\lambda_{1}\right)\left(1-\bar{m}_{A}(u)-\alpha\right) .
$$

Then, in view of the definition of $\Delta_{2}$, we have $\Delta_{2} \leq \alpha\left(u-\lambda_{1}\right)$, and

$$
\begin{aligned}
F_{2}\left(\Delta_{2}\right) & \leq\left(\sum_{i=1}^{n} \frac{1}{\left(u+\Delta_{2}-\lambda_{i}\right)\left(u-\lambda_{i}\right)}\right)^{-1} \sum_{i=1}^{n} \frac{\left\langle x, x_{i}\right\rangle^{2}}{\left(u-\lambda_{i}\right)^{2}} \\
& \leq(1+\alpha) F_{2}(0) .
\end{aligned}
$$

Hence,

$$
Q_{2}\left(\Delta_{2}\right)=F_{2}\left(\Delta_{2}\right) / \Delta_{2} \leq 1-\bar{m}_{A}(u)-\alpha
$$

If

$$
(1+\alpha) F_{2}(0)>\alpha\left(u-\lambda_{1}\right)\left(1-\bar{m}_{A}(u)-\alpha\right)
$$

then the statement follows directly from the definition of $\Delta_{2}$.

4.2. Randomization and control of expectations. In this subsection, we "randomize" Lemma 4.3 and Lemma 4.4. Let, as before, $A$ be an $n \times n$ (non-random) positive semidefinite matrix with eigenvalues $\lambda_{1} \geq \cdots \geq \lambda_{n} \geq 0$, let $u>\lambda_{1}$ satisfy $\bar{m}_{A}(u)<1$. We define (random) quantities $\Delta_{1}$ and $\Delta_{2}$ as in Lemma 4.3 and Lemma 4.4, replacing the fixed vector $x$ with a random isotropic vector $X$. In the following two lemmas, we will estimate the expectations of $\Delta_{1}$ and $\Delta_{2}$.

Lemma 4.5 (Control of $\left.\mathbb{E} \Delta_{1}\right)$. Let $\varepsilon \in(0,1 / 4]$ and let $A, \lambda_{i}, u$ be as above and $I_{j}$ be defined according to (4.2). Assume additionally that

$$
\max _{\ell \in \mathbb{N}} \frac{\left|I_{\ell}\right|}{16^{\ell}} \leq \frac{1}{\varepsilon n} .
$$

Further, let $X \in \mathbb{R}^{n}$ be an isotropic random vector and $K \geq 1$. Assume that for any non-zero orthogonal projection $\mathrm{P}: \mathbb{R}^{n} \rightarrow \mathbb{R}^{n}$ we have

$$
\mathbb{P}\left\{\|\mathrm{P}(X)\|^{2}-\operatorname{rankP} \geq t\right\} \leq \frac{g(\operatorname{rankP}) \operatorname{rankP}}{t^{2}}, t \geq f(\operatorname{rankP}) \operatorname{rankP},
$$

where functions $f: \mathbb{N} \rightarrow[0,1]$ and $g: \mathbb{N} \rightarrow[0, K]$ satisfy

$$
g(k) \leq \varepsilon^{11 / 2} \quad \text { and } \quad f(k) \leq \varepsilon^{2} \quad \forall k \geq \varepsilon^{17} n .
$$

Define a random variable $\Delta_{1}$ as in Lemma 4.3 replacing the non-random vector $x$ with $X$. Then

$$
\mathbb{E} \Delta_{1} \leq 32 K \sqrt{\varepsilon} .
$$

Proof. Let us estimate separately the expectations of $\Delta_{1}^{\prime}$ and $\Delta_{1, j},\left(j \leq \log _{4}\left(n / \varepsilon^{2}\right)\right)$, where the quantities are defined as in Lemma 4.3 . First,

$$
\begin{aligned}
\mathbb{E} \Delta_{1}^{\prime} & =\varepsilon^{-3 / 2} n \mathbb{P}\left\{\|X\|^{2} \geq n / \varepsilon\right\}+\int_{\varepsilon^{-3 / 2} n}^{\infty} \mathbb{P}\left\{\|X\|^{2} \geq \sqrt{\varepsilon} \tau\right\} d \tau \\
& \leq \frac{\varepsilon^{4}}{\left(\varepsilon^{-1}-1\right)^{2}}+\int_{\varepsilon^{-3 / 2} n-\varepsilon^{-1 / 2} n}^{\infty} \frac{\varepsilon^{9 / 2} n}{\tau^{2}} d \tau \\
& \leq \frac{\varepsilon^{4}}{\left(\varepsilon^{-1}-1\right)^{2}}+\frac{\varepsilon^{5}}{\varepsilon^{-1}-1} \\
& \leq 4 \varepsilon^{6} .
\end{aligned}
$$

Next, fix any natural $j \leq \log _{4}\left(n / \varepsilon^{2}\right)$ with $I_{j} \neq \varnothing$. We let $h_{j}$ to be defined as in (4.3), with the random vector $X$ replacing the non-random $x$. We have

$$
\mathbb{E} \Delta_{1, j}=\varepsilon 2^{j} \sqrt{\left|I_{j}\right|} \mathbb{P}\left\{h_{j}>\varepsilon^{2} 2^{j} \sqrt{\left|I_{j}\right|}\right\}+\int_{\varepsilon 2^{j} \sqrt{\left|I_{j}\right|}}^{\infty} \mathbb{P}\left\{h_{j} \geq \varepsilon \tau\right\} d \tau .
$$


Note that $h_{j}=\left\|\mathrm{P}_{j}(X)\right\|^{2}-\operatorname{rank} \mathrm{P}_{j}$ and $\left|I_{j}\right|=\operatorname{rankP}_{j}$, where $\mathrm{P}_{j}$ is the orthogonal projection onto the span of $\left\{x_{i}, i \in I_{j}\right\}$. Let us consider two cases.

1) $\left|I_{j}\right| \geq \varepsilon^{17} n$. Since $\bar{m}_{A}(u)<1$, we have $\left|I_{j}\right| \leq 4^{j}$, and $\varepsilon^{2}\left|I_{j}\right| \leq \varepsilon^{2} 2^{j} \sqrt{\left|I_{j}\right|}$. Hence, from the above formula for $\mathbb{E} \Delta_{1, j}$ and the conditions on projections of $X$ we obtain

$$
\begin{aligned}
\mathbb{E} \Delta_{1, j} & \leq \frac{\varepsilon^{5 / 2} \sqrt{\left|I_{j}\right|}}{2^{j}}+\frac{\varepsilon^{5 / 2} \sqrt{\left|I_{j}\right|}}{2^{j}} \\
& \leq 2^{j+1} \varepsilon^{5 / 2} \sqrt{\max _{\ell \in \mathbb{N}} \frac{\left|I_{\ell}\right|}{16^{\ell}}} \\
& \leq 2^{j+1} \varepsilon^{2} n^{-1 / 2} .
\end{aligned}
$$

2) $\left|I_{j}\right|<\varepsilon^{17} n$. Then, in view of Lemma 4.2, $\left|I_{j}\right| \leq \varepsilon^{8} 4^{j}$, implying

$$
f\left(\operatorname{rankP}_{j}\right) \leq 1 \leq\left|I_{j}\right|=\sqrt{\left|I_{j}\right|} \sqrt{\left|I_{j}\right|} \leq \varepsilon^{4} 2^{j} \sqrt{\left|I_{j}\right|}<\varepsilon^{2} 2^{j} \sqrt{\left|I_{j}\right|} .
$$

Applying again the formula for $\mathbb{E} \Delta_{1, j}$ together with the projection conditions and Lemma 4.2, we obtain

$$
\mathbb{E} \Delta_{1, j} \leq \frac{2 K \sqrt{\left|I_{j}\right|}}{\varepsilon^{3} 2^{j}} \leq \min \left(\frac{2^{j+1} K}{\varepsilon^{7 / 2} \sqrt{n}}, 2 \varepsilon K\right) .
$$

Thus, in any case $\mathbb{E} \Delta_{1, j} \leq 2^{j+1} \varepsilon^{2} n^{-1 / 2}+\min \left(\frac{2^{j+1} K}{\varepsilon^{7 / 2} \sqrt{n}}, 2 \varepsilon K\right)$. Summing over all $j \leq$ $\log _{4}\left(n / \varepsilon^{2}\right)$, we get

$$
\begin{aligned}
\sum_{j \leq \log _{4}\left(n / \varepsilon^{2}\right)} \mathbb{E} \Delta_{1, j} & \leq 4 \varepsilon+\sum_{j \leq \log _{4}\left(\varepsilon^{9} n\right)} \frac{2^{j+1} K}{\varepsilon^{7 / 2} \sqrt{n}}+2 K \varepsilon\left(-11 \log _{4} \varepsilon+1\right) \\
& \leq 4 \varepsilon+4 K \varepsilon+22 K \sqrt{\varepsilon}+2 K \varepsilon
\end{aligned}
$$

and the statement follows.

The next lemma does not require assumptions on projections of $X$ other than of rank one.

Lemma 4.6 (Control of $\left.\mathbb{E} \Delta_{2}\right)$. Let $A$ and $\lambda_{i}(i \leq n)$ be as above and let $X$ be an isotropic random vector in $\mathbb{R}^{n}$ such that for some $\kappa, K>0$ we have $\max _{\|y\|=1} \mathbb{E}|\langle X, y\rangle|^{2+\kappa} \leq K$, and let $u>\lambda_{1}$ and $\alpha \in(0,1 / 2]$ satisfy $\bar{m}_{A}(u)+\alpha<1$. Then for

$$
u\left[4.6:=K 2^{1+\kappa} /\left(\alpha^{1+\kappa / 2}\left(1-2^{-\kappa / 2}\right)\right)\right.
$$

we have

$$
\mathbb{E} \Delta_{2} \leq \frac{1+\alpha}{1-\bar{m}_{A}(u)-\alpha}\left(1+\frac{u_{4.6}}{\left(1-\bar{m}_{A}(u)-\alpha\right)^{\kappa / 2}\left(u-\lambda_{1}\right)^{\kappa / 2}}\right),
$$

where $\Delta_{2}$ is defined as in Lemma 4.4, with $X$ replacing $x$.

Proof. An inspection of the definition of $\Delta_{2}$ in Lemma 4.4 immediately shows that

$$
\Delta_{2} \leq \frac{(1+\alpha) F_{2}(0)}{1-\bar{m}_{A}(u)-\alpha}+\sum_{j=0}^{\infty} 2^{j}\left(u-\lambda_{1}\right) \chi_{j}
$$

where $\chi_{0}$ is the indicator function of the event

$$
\left\{(1+\alpha) F_{2}(0)>\alpha\left(u-\lambda_{1}\right)\left(1-\bar{m}_{A}(u)-\alpha\right)\right\}
$$

and for each $j>0, \chi_{j}$ is the indicator of the event

$$
\left\{Q_{2}\left(2^{j-1}\left(u-\lambda_{1}\right)\right)>1-\bar{m}_{A}(u)-\alpha\right\} .
$$


Note that for each fixed number $r \geq 0, F_{2}(r)$ is a random variable representable in the form

$$
F_{2}(r)=\sum_{i=1}^{n} \beta_{i}\left\langle X, x_{i}\right\rangle^{2},
$$

where the numbers $\beta_{i}=\beta_{i}(r)$ are positive and $\sum_{i=1}^{n} \beta_{i} \leq 1$. By the Minkowski inequality, for any $p \geq 1$ we have

$$
\left(\mathbb{E} F_{2}(r)^{p}\right)^{1 / p} \leq \sum_{i=1}^{n}\left(\mathbb{E}\left(\beta_{i}\left\langle X, x_{i}\right\rangle^{2}\right)^{p}\right)^{1 / p}=\sum_{i=1}^{n} \beta_{i}\left(\mathbb{E}\left|\left\langle X, x_{i}\right\rangle\right|^{2 p}\right)^{1 / p} \leq \max _{\|y\|=1}\left(\mathbb{E}|\langle X, y\rangle|^{2 p}\right)^{1 / p} .
$$

In particular, $\mathbb{E} F_{2}(r)^{1+\kappa / 2} \leq \max _{\|y\|=1} \mathbb{E}|\langle X, y\rangle|^{2+\kappa} \leq K$. Hence, from the definition of the indicator functions $\chi_{j}$ and applying Markov's inequality, we get

$$
\mathbb{E} \chi_{0} \leq K\left(\alpha(1+\alpha)^{-1}\left(u-\lambda_{1}\right)\left(1-\bar{m}_{A}(u)-\alpha\right)\right)^{-1-\kappa / 2},
$$

and for every $j \geq 1$ :

$$
\mathbb{E} \chi_{j} \leq K\left(2^{j-1}\left(u-\lambda_{1}\right)\left(1-\bar{m}_{A}(u)-\alpha\right)\right)^{-1-\kappa / 2} .
$$

Finally, we obtain

$$
\begin{aligned}
\mathbb{E} \Delta_{2} \leq & \frac{1+\alpha}{1-} \bar{m}_{A}(u)-\alpha \\
& +K\left(u-\lambda_{1}\right)\left(\alpha(1+\alpha)^{-1}\left(u-\lambda_{1}\right)\left(1-\bar{m}_{A}(u)-\alpha\right)\right)^{-1-\kappa / 2} \\
& +\sum_{j=1}^{\infty} 2^{j} K\left(u-\lambda_{1}\right)\left(2^{j-1}\left(u-\lambda_{1}\right)\left(1-\bar{m}_{A}(u)-\alpha\right)\right)^{-1-\kappa / 2} \\
= & \frac{1+\alpha}{1-\bar{m}_{A}(u)-\alpha} \\
& +\frac{K}{\left(1-\bar{m}_{A}(u)-\alpha\right)^{1+\kappa / 2}\left(u-\lambda_{1}\right)^{\kappa / 2}}\left(\frac{(1+\alpha)^{1+\kappa / 2}}{\alpha^{1+\kappa / 2}}+\sum_{j=1}^{\infty} 2^{1+\kappa / 2-\kappa j / 2}\right) \\
\leq & \frac{(1+\alpha)}{1-\bar{m}_{A}(u)-\alpha}\left(1+\frac{K(1+\alpha)^{\kappa / 2}}{\alpha^{1+\kappa / 2}\left(1-\bar{m}_{A}(u)-\alpha\right)^{\kappa / 2}\left(u-\lambda_{1}\right)^{\kappa / 2}} \sum_{j=0}^{\infty} 2^{1+\kappa / 2-\kappa j / 2}\right),
\end{aligned}
$$

and the result follows.

4.3. Proof of Theorem 1.7, completed. Let $X_{n}, m_{n}$ and $A_{n}, n \in \mathbb{N}$, be as in (1.1), (1.2), and (1.4) respectively. Without loss of generality, we can assume that both functions $f$ and $g$ in (STP) are non-increasing. Denote

$$
\gamma:=\inf _{n \rightarrow \infty} \frac{m_{n}}{n}>0
$$

and fix any $\varepsilon \in(0,1 / 16]$. We define $\alpha \in(0, \sqrt{\gamma} /(1+\sqrt{\gamma}))$ as the largest number satisfying

$$
\frac{1+\alpha}{1-t-\alpha} \leq \frac{1+\varepsilon}{1-t} \quad \text { for all } \quad t \in\left(0, \frac{1}{1+\sqrt{\gamma}}\right]
$$

Further, let $n_{\varepsilon} \in \mathbb{N}$ be such that

$$
g(k) \leq \varepsilon^{11 / 2} \quad \text { and } \quad f(k) \leq \varepsilon^{2} \quad \forall k \geq \varepsilon^{17} n_{\varepsilon}
$$

and, additionally,

$$
\frac{2(48+192 g(1)) \alpha^{-3 / 2}}{(\sqrt{\gamma} /(1+\sqrt{\gamma}))^{3 / 2}\left(\sqrt{\varepsilon n_{\varepsilon}} / 4\right)^{1 / 2}} \leq \varepsilon
$$


(the last condition will be needed later to simplify the estimate coming from Lemma 4.6). From now on, we fix $n \geq n_{\varepsilon}$. For convenience, we let $m:=m_{n}$ and $X^{(1)}, \ldots, X^{(m)}$ be i.i.d. copies of $X_{n}$. We define

$$
A^{(0)}:=0 \quad \text { and } \quad A^{(k)}=A^{(k-1)}+X^{(k)} \otimes X^{(k)}, \quad 1 \leq k \leq m,
$$

so that $A_{n}=A^{(m)}$. Set

$$
u_{0}:=n+\sqrt{m n}
$$

and let $u_{1}, \ldots, u_{m}$ be a collection of random numbers defined inductively as follows:

$$
u_{k}:=u_{k-1}+\Delta_{1}^{k}+\Delta_{2}^{k}+\Delta_{R}^{k},
$$

where $\Delta_{1}^{k}$ is taken from Lemma 4.3 (with $A^{(k-1)}, u_{k-1}$ and $X^{(k)}$ replacing $A, u$ and $x$, respectively), $\Delta_{2}^{k}$ is defined as in Lemma 4.4 (again, with $A^{(k-1)}, u_{k-1}$ and $X^{(k)}$ taking place of $A, u$ and $x$ ), and $\Delta_{R}^{k}$ is the regularity shift defined by

$\Delta_{R}^{k}:=\min \left\{\ell \in \mathbb{N} \cup\{0\}: \bar{m}_{A^{(k)}}\left(u_{k-1}+\Delta_{1}^{k}+\Delta_{2}^{k}+\ell\right)-\bar{m}_{A^{(k)}}\left(u_{k-1}+\Delta_{1}^{k}+\Delta_{2}^{k}+\ell+1\right) \leq \frac{1}{2 \varepsilon n}\right\}$

(the factor " 2 " in front of $\varepsilon n$ in the above definition does not carry any special meaning, and introduced for a purely technical reason). Note that, by lemmas 4.3 and 4.4, for any $k \geq 1$ we have $\bar{m}_{A^{(k)}}\left(u_{k-1}+\Delta_{1}^{k}+\Delta_{2}^{k}\right) \leq \bar{m}_{A^{(k-1)}}\left(u_{k-1}\right)$, implying

$$
\bar{m}_{A^{(k)}}\left(u_{k}\right)+\alpha \leq \bar{m}_{A^{(0)}}\left(u_{0}\right)+\alpha=\frac{n}{u_{0}}+\alpha=\frac{n}{n+\sqrt{m n}}+\alpha<1 \quad \text { (deterministically) }
$$

for all $k \geq 0$. The quantities $\Delta_{R}^{k}$ play a role analogous to numbers $\delta_{R}^{k}$ from the section dealing with the smallest eigenvalue. The following lemma gives an estimate of the cumulative impact of the regularity shifts:

Lemma 4.7. With $\Delta_{R}^{k}(1 \leq k \leq m)$ defined above, the following holds deterministically:

$$
\sum_{k=1}^{m} \Delta_{R}^{k} \leq 2 \varepsilon n
$$

Proof. Take any admissible $k \geq 1$. The definition of $\Delta_{R}^{k}$ immediately implies that for all $0 \leq \ell<\Delta_{R}^{k}$ we have

$$
\bar{m}_{A^{(k)}}\left(u_{k-1}+\Delta_{1}^{k}+\Delta_{2}^{k}+\ell\right)-\bar{m}_{A^{(k)}}\left(u_{k-1}+\Delta_{1}^{k}+\Delta_{2}^{k}+\ell+1\right)>\frac{1}{2 \varepsilon n} .
$$

Hence,

$$
\begin{aligned}
\bar{m}_{A^{(k)}}\left(u_{k}\right) & =\bar{m}_{A^{(k)}}\left(u_{k-1}+\Delta_{1}^{k}+\Delta_{2}^{k}+\Delta_{R}^{k}\right) \\
& \leq \bar{m}_{A^{(k)}}\left(u_{k-1}+\Delta_{1}^{k}+\Delta_{2}^{k}\right)-\frac{\Delta_{R}^{k}}{2 \varepsilon n} \\
& \leq \bar{m}_{A^{(k-1)}}\left(u_{k-1}\right)-\frac{\Delta_{R}^{k}}{2 \varepsilon n} .
\end{aligned}
$$

Thus, $\bar{m}_{A^{(k)}}\left(u_{k}\right) \leq \bar{m}_{A^{(k-1)}}\left(u_{k-1}\right)-\frac{\Delta_{k}^{k}}{2 \varepsilon n}$ for all $k \geq 1$, which, together with the relations $0<\bar{m}_{A^{(k)}}\left(u_{k}\right)<1(1 \leq k \leq m)$, implies the result.

Now, fix for a moment any $k \in\{1, \ldots, m\}$ and let $\mathcal{F}_{k-1}$ be the $\sigma$-algebra generated by the vectors $X^{(1)}, \ldots, X^{(k-1)}$. We will first estimate the conditional expectation

$$
\mathbb{E}\left(\Delta_{1}^{k}+\Delta_{2}^{k} \mid \mathcal{F}_{k-1}\right)=\mathbb{E}\left(\Delta_{1}^{k} \mid \mathcal{F}_{k-1}\right)+\mathbb{E}\left(\Delta_{2}^{k} \mid \mathcal{F}_{k-1}\right) .
$$

Let the sets $I_{j}(j \in \mathbb{N})$ be defined by (4.2) with $A^{(k-1)}$ and $u_{k-1}$ replacing $A$ and $u$, i.e.

$$
I_{j}:=\left\{i \leq n: 4^{j-1} \leq u_{k-1}-\lambda_{i}\left(A^{(k-1)}\right)<4^{j}\right\}, \quad j \in \mathbb{N} .
$$


Note that the summand $\Delta_{R}^{k-1}$ makes sure that

$$
\sum_{j=1}^{\infty} \frac{\left|I_{j}\right|}{16^{j}} \leq \sum_{j=1}^{\infty} \frac{2\left|I_{j}\right|}{4^{j}\left(4^{j}+1\right)}<2\left(\bar{m}_{A^{(k-1)}}\left(u_{k-1}\right)-\bar{m}_{A^{(k-1)}}\left(u_{k-1}+1\right)\right) \leq \frac{1}{\varepsilon n}
$$

(when $k=1$, the above estimate holds as well since in that case $\sum_{j=1}^{\infty} \frac{\left|I_{j}\right|}{16^{j}} \leq \frac{16 n}{u_{0}^{2}}<\frac{1}{\varepsilon n}$ ). Applying Lemma 4.5, we obtain

$$
\left\|\mathbb{E}\left(\Delta_{1}^{k} \mid \mathcal{F}_{k-1}\right)\right\|_{\infty} \leq 32 g(1) \sqrt{\varepsilon} .
$$

Next, note that the assumptions on 1-dimensional projections imply that for any unit vector $y$ we have

$$
\begin{aligned}
\mathbb{E}\left|\left\langle y, X_{n}\right\rangle\right|^{3} & =\int_{0}^{\infty} \mathbb{P}\left\{\left\langle y, X_{n}\right\rangle^{2} \geq \tau^{2 / 3}\right\} d \tau \\
& \leq \sqrt{8}+\int_{\sqrt{8}}^{\infty} \mathbb{P}\left\{\left\langle y, X_{n}\right\rangle^{2}-1 \geq \tau^{2 / 3}-1\right\} d \tau \\
& \leq \sqrt{8}+4 g(1) \int_{\sqrt{8}}^{\infty} \tau^{-4 / 3} d \tau \\
& \leq \sqrt{8}+12 g(1) .
\end{aligned}
$$

Next, the upper bound on $\frac{\left|I_{j}\right|}{16^{j}}$ implies that $I_{j}=\varnothing$ whenever $j \leq \log _{16}(\varepsilon n)$, whence

$$
u_{k-1}-\lambda_{1}\left(A^{(k-1)}\right) \geq \frac{\sqrt{\varepsilon n}}{4} .
$$

Then, by Lemma 4.6 (applied with $\kappa:=1$ ), and in view of the inequality $\bar{m}_{A^{(k-1)}}\left(u_{k-1}\right) \leq$ $\bar{m}_{A^{(0)}}\left(u_{0}\right)$, we get

$$
\begin{aligned}
& \left\|\mathbb{E}\left(\Delta_{2}^{k} \mid \mathcal{F}_{k-1}\right)\right\|_{\infty} \\
& \quad \leq \frac{1+\alpha}{1-\bar{m}_{A^{(0)}}\left(u_{0}\right)-\alpha}\left(1+\frac{u_{4.6}}{\left(1-\bar{m}_{A^{(0)}}\left(u_{0}\right)-\alpha\right)^{1 / 2}(\sqrt{\varepsilon n} / 4)^{1 / 2}}\right),
\end{aligned}
$$

where

$$
u\left[4.6=4 \sup _{\|y\|=1} \mathbb{E}\left|\left\langle y, X_{n}\right\rangle\right|^{3} /\left(\alpha^{3 / 2}\left(1-2^{-1 / 2}\right)\right) \leq(48+192 g(1)) \alpha^{-3 / 2} .\right.
$$

By the choice of $\alpha$, we have

$$
\frac{1+\alpha}{1-\bar{m}_{A^{(0)}}\left(u_{0}\right)-\alpha} \leq \frac{1+\varepsilon}{1-\bar{m}_{A^{(0)}}\left(u_{0}\right)},
$$

and by the choice of $n_{\varepsilon}$, we have

$$
\frac{u_{4.6}(1+\alpha)}{\left(1-\bar{m}_{A^{(0)}}\left(u_{0}\right)-\alpha\right)^{3 / 2}(\sqrt{\varepsilon n} / 4)^{1 / 2}} \leq \varepsilon .
$$

Hence, we obtain

$$
\left\|\mathbb{E}\left(\Delta_{2}^{k} \mid \mathcal{F}_{k-1}\right)\right\|_{\infty} \leq(1+\varepsilon)\left(1+\sqrt{\frac{n}{m}}\right)+\varepsilon .
$$

Summing up over $k \in\{1, \ldots, m\}$ and applying Lemma 4.7, we get

$$
\begin{aligned}
\mathbb{E} \lambda_{1}\left(A_{n}\right) & \leq \mathbb{E} u_{m} \\
& \leq n+\sqrt{m n}+32 g(1) \sqrt{\varepsilon} m+(1+\varepsilon)(m+\sqrt{m n})+\varepsilon m+2 \varepsilon n \\
& =(\sqrt{n}+\sqrt{m})^{2}+32 g(1) \sqrt{\varepsilon} m+\varepsilon(2 m+2 n+\sqrt{m n}) .
\end{aligned}
$$

Since $\varepsilon$ can be chosen arbitrarily small, we get the result. 


\section{ACKNOWLEDGEMENTS}

D.C. would like to warmly thank Radosław Adamczak, Charles Bordenave, and Alain Pajor for discussions on this topic in Warsaw, Toulouse, and Paris. K.T. would like to thank Pierre Youssef for introducing him to the result of Batson-Spielman-Srivastava and especially thank Prof. Alain Pajor for the invitation to visit UPEM in NovemberDecember, 2014. A significant part of the present work was done during that period.

\section{REFERENCES}

[ABP03] Milla Anttila, Keith Ball, and Irini Perissinaki, The central limit problem for convex bodies, Trans. Amer. Math. Soc. 355 (2003), no. 12, 4723-4735 (electronic). MR-1997580 7

[AC15] Radosław Adamczak and Djalil Chafaï, Circular law for random matrices with unconditional log-concave distribution, Commun. Contemp. Math. 17 (2015), no. 4, 1550020 (22 pages). MR-3359233 3

[Ada11] Radosław Adamczak, On the Marchenko-Pastur and circular laws for some classes of random matrices with dependent entries, Electron. J. Probab. 16 (2011), no. 37, 1068-1095. MR-2820070 [3]

$\left[\mathrm{ALL}^{+} 14\right]$ Radosław Adamczak, Rafał Latała, Alexander E. Litvak, Krzysztof Oleszkiewicz, Alain Pajor, and Nicole Tomczak-Jaegermann, A short proof of Paouris' inequality, Canad. Math. Bull. 57 (2014), no. 1, 3-8. MR-3150710[7

[ALPTJ10] Radosław Adamczak, Alexander E. Litvak, Alain Pajor, and Nicole Tomczak-Jaegermann, Quantitative estimates of the convergence of the empirical covariance matrix in log-concave ensembles, J. Amer. Math. Soc. 23 (2010), no. 2, 535-561. MR-2601042 3

[ALPTJ11] _ Sharp bounds on the rate of convergence of the empirical covariance matrix, C. R. Math. Acad. Sci. Paris 349 (2011), no. 3-4, 195-200. MR-2769907 3

[BF03] Alexei Borodin and Peter J. Forrester, Increasing subsequences and the hard-to-soft edge transition in matrix ensembles, J. Phys. A 36 (2003), no. 12, 2963-2981, Random matrix theory. MR-1986402 7

[BGN12] Florent Benaych-Georges and Raj Rao Nadakuditi, The singular values and vectors of low rank perturbations of large rectangular random matrices, J. Multivariate Anal. 111 (2012), 120-135. MR-2944410[3]

[BGVV14] Silouanos Brazitikos, Apostolos Giannopoulos, Petros Valettas, and Beatrice-Helen Vritsiou, Geometry of isotropic convex bodies, Mathematical Surveys and Monographs, vol. 196, American Mathematical Society, Providence, RI, 2014. MR-3185453 2

[Bor74] Christer Borell, Convex measures on locally convex spaces, Ark. Mat. 12 (1974), 239-252. MR-03884752

[Bor14] Charles Bordenave, Notes on random matrices, available on the Internet, 2014. 3

[BS98] Zhi-Dong Bai and Jack W. Silverstein, No eigenvalues outside the support of the limiting spectral distribution of large-dimensional sample covariance matrices, Ann. Probab. 26 (1998), no. 1, 316-345. MR-1617051 3] 7

[BS10] Spectral analysis of large dimensional random matrices, second ed., Springer Series in Statistics, Springer, New York, 2010. 3

[BS12] No eigenvalues outside the support of the limiting spectral distribution of informationplus-noise type matrices, Random Matrices Theory Appl. 1 (2012), no. 1, 1150004, 44. MR-2930382 7

[BSS09] Joshua D. Batson, Daniel A. Spielman, and Nikhil Srivastava, Twice-Ramanujan sparsifiers, STOC'09-Proceedings of the 2009 ACM International Symposium on Theory of Computing, ACM, New York, 2009, pp. 255-262. MR-2780071 3, 6]

[BSS12] Joshua Batson, Daniel A. Spielman, and Nikhil Srivastava, Twice-Ramanujan sparsifiers, SIAM J. Comput. 41 (2012), no. 6, 1704-1721. MR-3029269 3, 13, 18

[BY88] Zhi-Dong Bai and Yong-Qua Yin, Necessary and sufficient conditions for almost sure convergence of the largest eigenvalue of a Wigner matrix, Ann. Probab. 16 (1988), no. 4, 1729-1741. 3

[BY93] Limit of the smallest eigenvalue of a large-dimensional sample covariance matrix, Ann. Probab. 21 (1993), no. 3, 1275-1294. MR-1235416 3

[BZ08] Zhi-Dong Bai and Wang Zhou, Large sample covariance matrices without independence structures in columns, Statist. Sinica 18 (2008), no. 2, 425-442. MR-2411613 3

[CDM07] Mireille Capitaine and Catherine Donati-Martin, Strong asymptotic freeness for Wigner and Wishart matrices, Indiana Univ. Math. J. 56 (2007), no. 2, 767-803. 3 
[dlPG99] Víctor H. de la Peña and Evarist Giné, Decoupling, Probability and its Applications (New York), Springer-Verlag, New York, 1999, From dependence to independence, Randomly stopped processes. $U$-statistics and processes. Martingales and beyond. MR-1666908 [10]

[Fel71] William Feller, An introduction to probability theory and its applications. Vol. II., Second edition, John Wiley \& Sons, Inc., New York-London-Sydney, 1971. MR-02704038

[Fle10] Bruno Fleury, Concentration in a thin Euclidean shell for log-concave measures, J. Funct. Anal. 259 (2010), no. 4, 832-841. MR-2652173 7

[FS10] Ohad N. Feldheim and Sasha Sodin, A universality result for the smallest eigenvalues of certain sample covariance matrices, Geom. Funct. Anal. 20 (2010), no. 1, 88-123. MR-2647136 7

[GM11] Olivier Guédon and Emanuel Milman, Interpolating thin-shell and sharp large-deviation estimates for isotropic log-concave measures, Geom. Funct. Anal. 21 (2011), no. 5, 1043-1068. MR-2846382 7

[Gué14] Olivier Guédon, Concentration phenomena in high dimensional geometry, Journées MAS 2012, ESAIM Proc., vol. 44, EDP Sci., Les Ulis, 2014, pp. 47-60. MR-3178607[7]

[HT05] Uffe Haagerup and Steen Thorbjørnsen, A new application of random matrices: $\operatorname{Ext}\left(C_{\mathrm{red}}^{*}\left(F_{2}\right)\right)$ is not a group, Ann. of Math. (2) 162 (2005), no. 2, 711-775. MR-21832813

[Joh00] Kurt Johansson, Shape fluctuations and random matrices, Comm. Math. Phys. 209 (2000), no. 2, 437-476. MR-17379910

[Joh01] Iain M. Johnstone, On the distribution of the largest eigenvalue in principal components analysis, Ann. Statist. 29 (2001), no. 2, 295-327. MR-1863961 7

[Kla07] B. Klartag, Power-law estimates for the central limit theorem for convex sets, J. Funct. Anal. 245 (2007), no. 1, 284-310. MR-2311626 7

[KM15] Vladimir Koltchinskii and Shahar Mendelson, Bounding the smallest singular value of a random matrix without concentration, preprint arXiv:1312.3580 to appear in International Mathematics Research Notices, 2015. 3

[LY14] Ji Oon Lee and Jun Yin, A necessary and sufficient condition for edge universality of Wigner matrices, Duke Math. J. 163 (2014), no. 1, 117-173. MR-3161313 7]

[MP67] Vladimir O. Marchenko and Leonid A. Pastur, Distribution of eigenvalues in certain sets of random matrices, Mat. Sb. (N.S.) 72 (114) (1967), 507-536. 2

[Pao06] Grigoris Paouris, Concentration of mass on convex bodies, Geom. Funct. Anal. 16 (2006), no. 5, 1021-1049. MR-2276533 7

[Péc09] Sandrine Péché, Universality results for the largest eigenvalues of some sample covariance matrix ensembles, Probab. Theory Related Fields 143 (2009), no. 3-4, 481-516. MR-2475670. 7

[PP09] Alain Pajor and Leonid A. Pastur, On the limiting empirical measure of eigenvalues of the sum of rank one matrices with log-concave distribution, Studia Math. 195 (2009), no. 1, 1129. MR-25395592

[PS11] Leonid A. Pastur and Mariya Shcherbina, Eigenvalue distribution of large random matrices, Mathematical Surveys and Monographs, vol. 171, American Mathematical Society, Providence, RI, 2011. MR-2808038 2, 3

[PY14] Natesh S. Pillai and Jun Yin, Universality of covariance matrices, Ann. Appl. Probab. 24 (2014), no. 3, 935-1001. MR-3199978 3 7

[RG14] Kevin Richard and Alice Guionnet, Central limit theorem and convergence of the support for Wishart matrices with correlated entries, preprint arXiv:1401.7367, 2014. 3

[RV10] Mark Rudelson and Roman Vershynin, Non-asymptotic theory of random matrices: extreme singular values, Proceedings of the International Congress of Mathematicians. Volume III, Hindustan Book Agency, New Delhi, 2010, pp. 1576-1602. MR-2827856 3

[Sch05] Hanne Schultz, Non-commutative polynomials of independent Gaussian random matrices. The real and symplectic cases, Probab. Theory Related Fields 131 (2005), no. 2, 261-309. 3

[Sos02] Alexander Soshnikov, A note on universality of the distribution of the largest eigenvalues in certain sample covariance matrices, J. Statist. Phys. 108 (2002), no. 5-6, 1033-1056, Dedicated to David Ruelle and Yasha Sinai on the occasion of their 65th birthdays. MR-1933444 7

[SV13] Nikhil Srivastava and Roman Vershynin, Covariance estimation for distributions with $2+\varepsilon$ moments, Ann. Probab. 41 (2013), no. 5, 3081-3111. MR-3127875 3, 4, 6, 10, 13, 18, 19,

[Tik15] Konstantin Tikhomirov, The limit of the smallest singular value of random matrices with i.i.d. entries, Adv. Math. 284 (2015), 1-20. 3, 6, 10

[Ver11] Roman Vershynin, A simple decoupling inequality in probability theory, unpublished, available on the Internet, 2011. 10

[Yas14] Pavel Yaskov, Lower bounds on the smallest eigenvalue of a sample covariance matrix, Electron. Commun. Probab. 19 (2014), no. 83, 1-10. 3 
[Yas15] Sharp lower bounds on the least singular value of a random matrix without the fourth moment condition, Electron. Commun. Probab. 20 (2015), no. 44, 1-9. 3

[YBK88] Yong-Qua Yin, Zhi-Dong Bai, and Paruchuri R. Krishnaiah, On the limit of the largest eigenvalue of the large-dimensional sample covariance matrix, Probab. Theory Related Fields $\mathbf{7 8}$ (1988), no. 4, 509-521. MR-9503443

(DC) Ceremade, Université Paris-Dauphine, PSL, iUf, Paris, France.

(KT) Dep. of Math. and Stat. Sciences, University of Alberta, Edmonton, Canada. 